\title{
An Actuator with Mechanically Adjustable Series Compliance
}

Jonathan W. Hurst

Joel E. Chestnutt

June 13, 2005

CMU-RI-TR-04-24
Alfred A. Rizzi

\author{
April 2004 \\ Robotics Institute \\ Carnegie Mellon University \\ Pittsburgh, Pennsylvania 15213 \\ (c) Carnegie Mellon University
}




\begin{abstract}
Running is a complex dynamic task which places strict requirements on both the physical components and software control systems of a robot. This report explores some of those requirements and in particular explores how a variable compliance actuation system can satisfy many of them. We present the mechanical design and software control of such an actuator system. We analyze its performance through simulation and benchtop experimental validation of a prototype version. In conclusion we demonstrate, through simulation, the application of our prototype actuator to the problem of biped running.
\end{abstract}




\section{Contents}

1 Introduction

2 Background

2.1 Compliance and Running . . . . . . . . . . . . . . . . . . . . . . . . . . 2

2.2 Physically Variable Compliance as a Method of Control . . . . . . . . . . . . . . . . . . . . . . 2

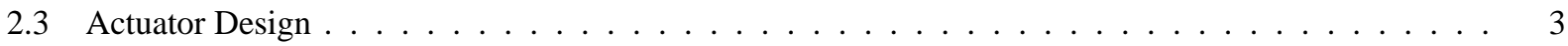

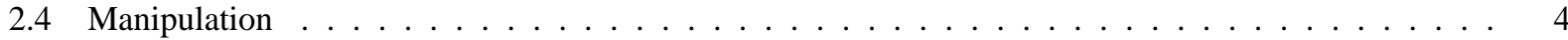

3 The AMASC Design 4

3.1 Mechanical Design . . . . . . . . . . . . . . . . . . . . . . . . 4

3.2 Control System Design . . . . . . . . . . . . . . . . . . . . . . . 9

4 Simulation, Results and Comparison $\quad 10$

4.1 Static Compliance Characterization . . . . . . . . . . . . . . . . . . . . . . 10

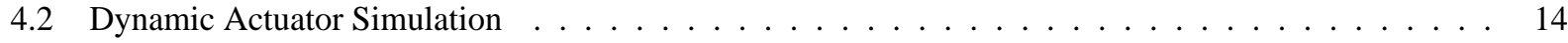

4.3 Running Simulation . . . . . . . . . . . . . . . . . . . . . . . . . 16

5 Discussion $\quad \mathbf{1 6}$

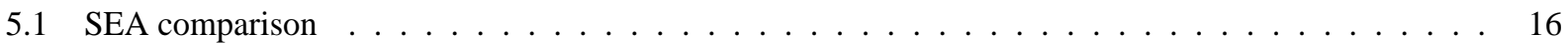

5.2 The AMASC as a Manipulator . . . . . . . . . . . . . . . . . . . 20

6 Conclusions $\quad \mathbf{2 0}$

6.1 Future Work . . . . . . . . . . . . . . . . . . . . . . . . . . 20 


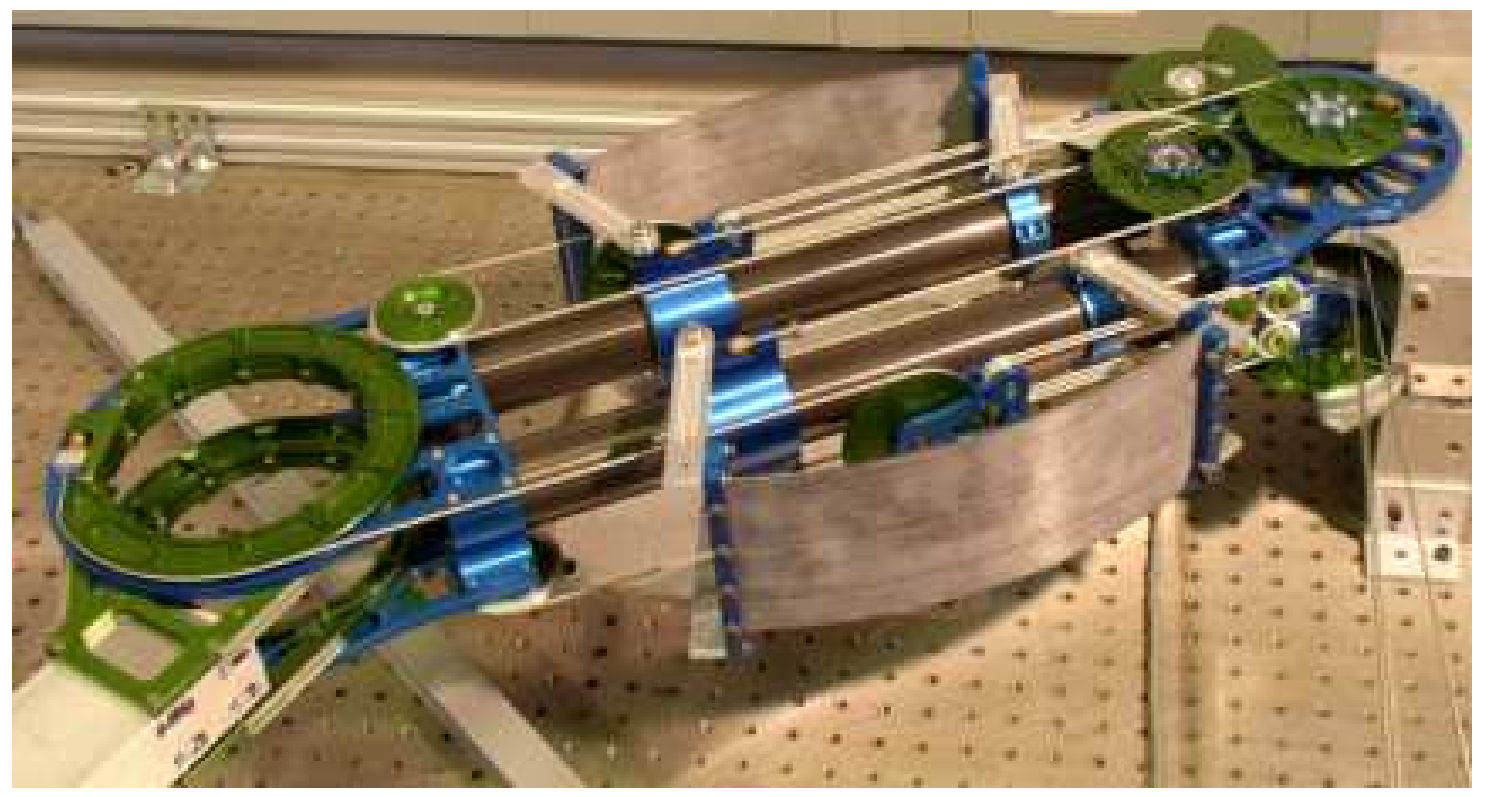

Figure 1: An Actuator with Mechanically Adjustable Series Compliance (AMASC)

\section{Introduction}

A robot designed specifically for autonomous legged locomotion should be capable of highly dynamic running, jumping, and stumble recovery, and should achieve these capabilities while being energetically efficient. In order to accomplish these goals, we believe a leg spring of sufficient capacity to store the energy of a running gait is virtually a necessity. Furthermore, we believe variable leg spring stiffness provides an important means for effective gait control.

One could conceive of two extremes of actuator design that would allow the desired capabilities. The first extreme utilizes high-bandwidth actuators, with all dynamic properties described by software control policies. The second extreme uses a carefully designed mechanical system with tuned natural dynamics that require no software control. The first method is flexible, although actuators with sufficiently high bandwidth and power capacity may not exist for the locomotion task, limiting behavior to low-frequency situations. The second method is quite inflexible, and requires extensive knowledge of the desired behavior before construction. However, there are no bandwidth limits preventing the mechanism from behaving as intended at high frequencies. In addition, this method is potentially very energy-efficient, as it takes full advantage of natural dynamics in achieving its goal. This method is task-specific, with natural dynamics that are applicable in only a small number of situations. Resulting designs are unlikely to be able to perform the breadth of tasks required for running.

Our design, shown in Figures 1 and 5, represents a carefully chosen balance between the two design extremes. The natural dynamics of the system are engineered and utilized to where possible, while the software control system adds energy that is lost in the mechanism, and creates behaviors that are not inherent in the natural dynamics. This Actuator with Mechanically Adjustable Series Compliance (AMASC) exhibits natural dynamics that are similar to those of animals, and is a mechanical instantiation of a previously developed mathematical model of running, shown in Figure 2, the spring loaded inverted pendulum (SLIP) [1, 2, 3].

\section{Background}

Most research papers that analyze the mechanics of running base this analysis on some form of the SLIP model, shown in Figure 2. This model can be used to generate the motion of the center of mass of a running animal $[4,5]$ or a running robot. The basic definition of running [6] is linked to the SLIP idea - energy is transferred from kinetic and gravitational energy in the flight phase to spring energy in the stance phase, and vice versa. The natural physical instantiation of the SLIP model utilizes passive leg springs for this energy storage. 


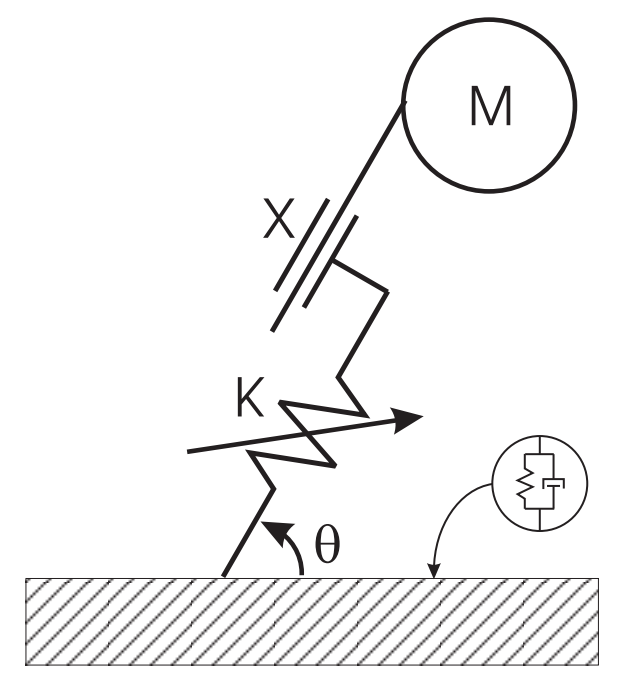

Figure 2: The Spring Loaded Inverted Pendulum Model can control a running gait by manipulation of three variables: a spring constant $K$, spring rest length $x$, and touchdown angle $\theta$. Properties of the ground also affect the dynamics of the SLIP model.

\subsection{Compliance and Running}

Physical series compliance is virtually necessary to achieve a successful running gait. Simulating compliance using a rigid actuator such as an electric gearmotor is not feasible for three reasons: bandwidth limitations, power output limitations, and energetic efficiency. The bandwidth limitation of an electric motor is due, in large part, to the high reflected inertia linked rigidly to the robot leg. Electric motors must be significantly geared to provide useful torque for robotics applications, and the reflected inertia of the rotor is amplified by the square of the gear reduction. The reflected inertia can be larger than the inertia of the entire robot, making a correct dynamic response to impacts impossible. Even with instantaneous sensing and computation, the torque required to accelerate the motor instantly to match the ground speed during an unexpected impact is far greater than can be achieved with an electric motor.

Passive series springs can alleviate many of the problems inherent in gearmotors. They can be used for energy storage, power transmission, or a combination of the two, as illustrated in Figure 3. The power density of a physical spring is arbitrarily high, depending on its stiffness. Motors and batteries have relatively low power density in comparison, but have a much higher work capacity per unit mass, making a compelling argument for combining the two systems in a series combination. The spring can absorb and output large amounts of energy in short bursts and at high power, while the motor can add and remove energy as needed, without handling all of the power transferred in a running gait.

Springs are particularly useful in rhythmic systems, because energy can be stored and released much more efficiently through a spring than if it were passed through the motor, transmission, and power electronics with each transfer. Not only do physical springs reduce wasted energy with each stride, but they also significantly reduce the necessary size and weight of many of the motor and transmission components. Minimizing weight reduces the total amount of energy that needs to be transferred with each stride. Animals store mechanical spring energy during a running gait, most likely for these reasons and more $[7,8,9,10]$.

\subsection{Physically Variable Compliance as a Method of Control}

While physical compliance is virtually a necessity for successful running, varying the compliance provides a useful tool for gait control. Animals have physically variable leg compliance (tunable natural dynamics), and vary it to control running and hopping in certain situations $[3,4,11,12,13,14,15,16]$.

Leg stiffness is one of three terms available to the SLIP model for control of a running gait, along with hip angle and spring set point. The normal steady-state motion of a SLIP-based running gait can be described by three parameters, which will be influenced by the leg stiffness. For the mathematical analysis performed by Schwind and Koditschek [1], leg length and angular velocity at bottom of stance along with the leg stiffness were chosen as the three parameters. Control of forward speed, stance duration, and flight duration was demonstrated experimentally 


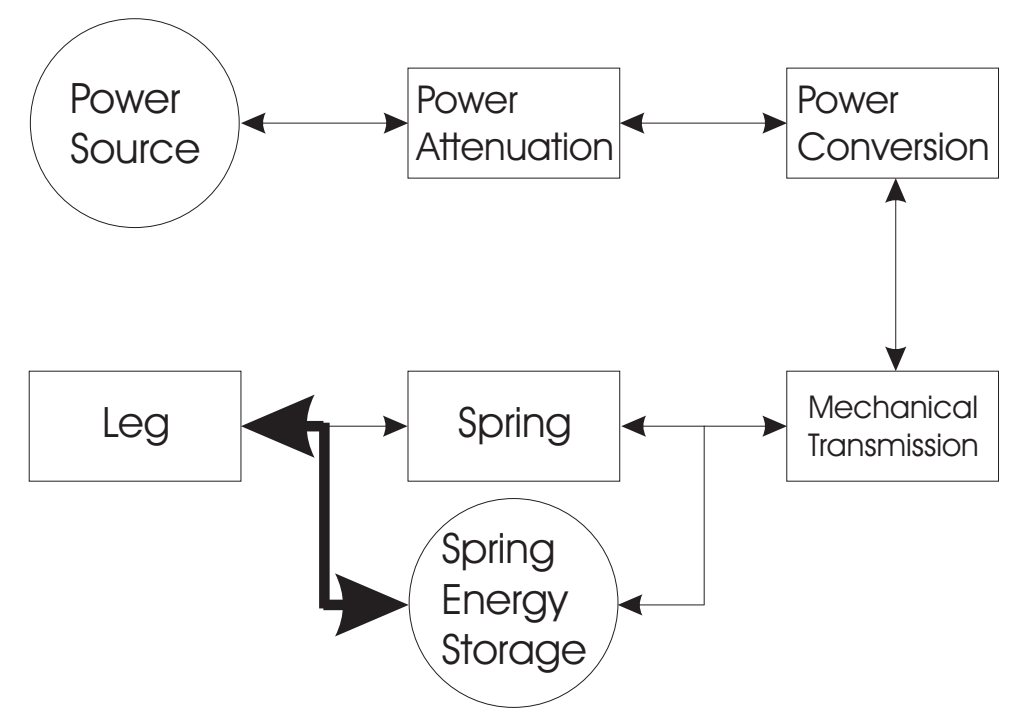

Figure 3: Most of the energy of a running gait can be stored and returned with each stride, with a properly sized and tuned mechanical spring.

in [17]. Another choice might be leg stiffness, hopping height, and stride length; there are many possible 3-variable parameterizations that could be considered. Because the SLIP model has only three terms available to control the three parameters of a running gait, the absence of one term may limit the control choices, and certain disturbances to the SLIP model running gait may not be controllable. For example, if a SLIP runs over terrain that varies in stiffness, the leg stiffness must change or the running gait will be altered.

Global vertical stiffness (GVS) is a useful concept when discussing a running gait, and is calculated by considering only the vertical excursion of the center of mass. The GVS is influenced by changes in leg stiffness[12], touchdown angle, ground stiffness, and the number of legs on the ground at once. In observations of animal running, the GVS increases with the square of the running speed [16, 3], implying that the GVS is a direct method of control for forward running speed. Most research suggests that animals prefer to maintain leg stiffness over a range of running speeds $[3,13,18,19]$, using other methods to change global vertical stiffness; however, they do change leg stiffness when other methods are not available. For example, hopping or running on a surface of changing stiffness [4, 11, 13], hopping in place with varying frequency $[4,11]$, or running at different speeds with constant stride length [18].

Previous successful running robots have physical compliance tuned for a hard laboratory floor, but do not attempt to run on terrains of varying stiffness. Therefore, they do not require the ability to vary their leg stiffness to maintain a tuned SLIP-model running gait. If one of these running robots unexpectedly began running on a soft ground surface, the gait would be changed in a manner that the controller would be unable to rectify. This gait change may or may not cause the robot to fall, depending on the severity of the change; but it would slow down or speed up the forward speed, or change the hopping height, or change the stride length. For future robots intended to run over various ground surfaces, stiffness variation will be a useful control parameter.

\subsection{Actuator Design}

Methods of actuation other than gearmotors have been used for legged locomotion. Hydraulic actuators have many of the same limitations of gearmotors, and most arguments regarding gearmotors can also be applied to hydraulic actuators. Pneumatic actuators are inherently springy, which has led to their successful use on several running and hopping robots. However, pneumatic actuators are also inherently difficult to control, energetically lossy, and difficult to power without an external compressor. Electrical actuation allows for much more precise control, and allows for easier tether-free operation. For these reasons, we chose to use electric actuation, although the ideas presented in this paper could be implemented with some other form of actuation.

Researchers at MIT developed a Series Elastic Actuator (SEA) that used both electric and hydraulic actuation[20]. The primary differences between the SEA and the AMASC are that the mechanical springs are dramatically different in size, and the AMASC can vary its stiffness while the SEA cannot. The SEA was developed as a force actuator for 
robot arms, and the design was later refined and used on several walking robots [21, 22, 23, 24].

\subsection{Manipulation}

Although the AMASC is being developed for legged locomotion, the mechanism is also applicable to manipulation tasks. For example, variable stiffness is useful for relatively high-performance, safe robot-human interaction[25]. While the robot is moving slowly, the arm can have very stiff joints for improved positional accuracy, and a collision will result in a relatively low impact force. When the arm moves faster, the stiffness of the transmission must decrease, so the inertia of the motor is disconnected from the arm, and an arm impact will still result in a relatively low impact force.

Mechanically adjustable series compliance may also be useful for control stability. Several researchers have shown that certain types of physical compliance is important for force control [26, 27, 28]; it is intuitive that a very lowstiffness joint is more capable of applying a constant force in the face of position disturbances than a rigid joint. For different force control tasks, different joint stiffnesses may be desirable. Humans utilize variable joint stiffness for reaching motions[29]. When holding the hand at a particular position, humans exhibit less tremor with low joint stiffness, indicating better control stability; however, an outside force disturbance is more likely to displace the hand[30]. Thus, joint stiffness can be varied in a trade-off between disturbance rejection and control stability.

\section{The AMASC Design}

The natural dynamics of a system are an inseparable part of its behavior. The mechanical design, which determines the natural dynamics of a system, is thus an essential part of the overall control system design. The actuator presented here is an integrated mechanism and software controller, with mechanical design choices made to closely match a simple mechanical model. The software controller is based on the same simple mechanical model, safely ignoring most of the complexities of the actual mechanism. This model is illustrated in Figure 4, in two different forms: one rotational, one linear.

The rotational model is physically similar to the prototype AMASC, while the linear model is a simpler form that still captures the important properties of the system. In both models, the dynamics of the system controlling the pretension, $x_{3}$, are ignored. The pretension is entirely unrepresented in the linear model, and the spring stiffness, $K_{\text {eff }}$, is assumed to simply be a programmable value. Throughout the remainder of this paper we will use the notational conventions of Table 1.

The AMASC is essentially a single compliant joint, most closely resembling a knee, endowed with engineered natural dynamics. There are two degrees of freedom, and two corresponding motors. One motor controls the spring pretension. As shown in Figure 4(a), there are two identical opposing springs, much like antagonistic muscles in animals. The pretension, $x_{3}$, stretches both springs, which is analogous to muscle co-contraction in animals. The knee joint does not move, but its rotational stiffness increases with the pretension, allowing the actuator to tune the stiffness aspect of its natural dynamics. The other motor controls the spring rest position $\left(\theta_{1}\right.$ in Figure 4(a) and $x_{1}$ in Figure 4(b)), which is used as the primary energy source and controls any motions not described by the system's natural dynamics. These two motor-controlled parameters, along with the leg angle at touchdown, are the parameters necessary to control SLIP model running.

\subsection{Mechanical Design}

Many of the mechanism design challenges are common ones; for example, minimizing friction, backlash, mass, and inertia. Several specific choices were made that influence each of these attributes, such as the location and type of speed reduction, choice of materials, and type of motor. Minimizing mass was a concern throughout the design process, because the AMASC is intended as a prototype leg for a bipedal robot with approximately 1 meter leg length and $30 \mathrm{~kg}$ total mass. Of this $30 \mathrm{~kg}, 20 \mathrm{~kg}$ are reserved for motors, batteries, and computing. This allotment leaves only $10 \mathrm{~kg}$ for the entire framework and mechanism, including springs and power transmission. To minimize weight, all joints contain thin-section bearings, which are very light for a given load rating. All parts are machined aluminum, with the main structural members (analogous to the femur) made of thin-wall aluminum tube. The mass of this actuator prototype is approximately $4 \mathrm{~kg}$, and it is nearly $50 \%$ oversized. 


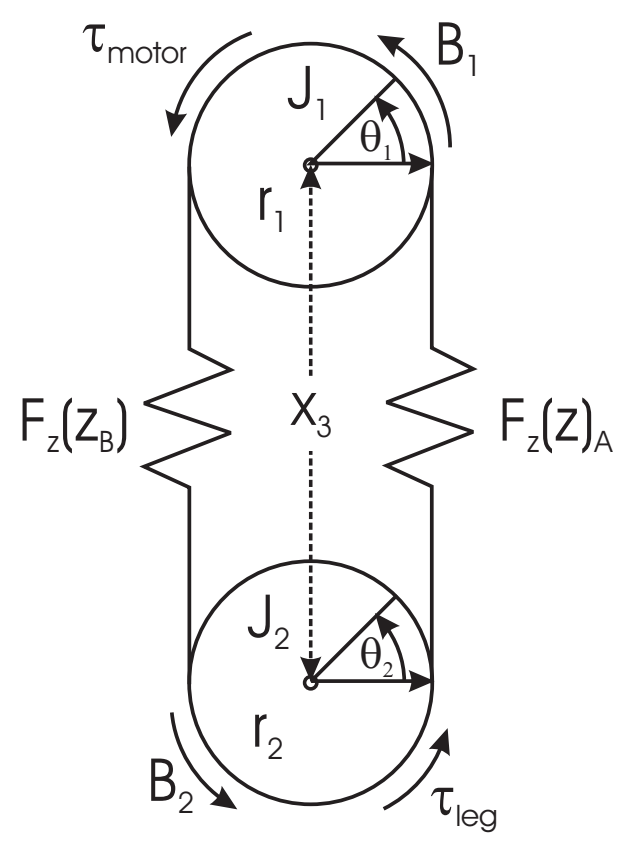

(a) Rotational model

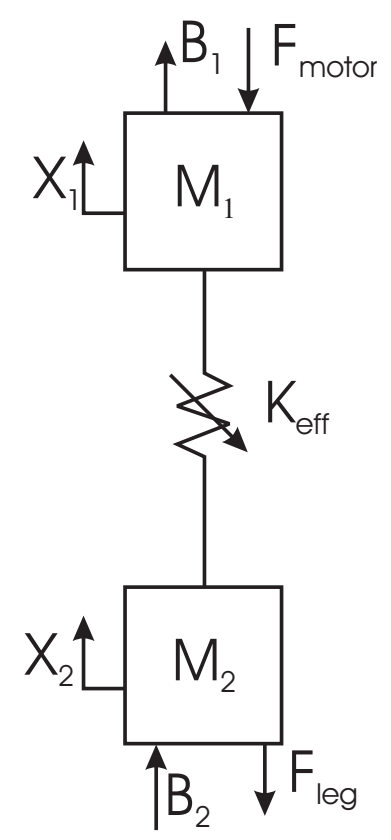

(b) Linear model

Figure 4: Mechanical models of the physical actuator

\begin{tabular}{|r|l|}
\hline variable & description \\
\hline \hline$\theta_{1}$ & Motor position \\
\hline$\theta_{2}$ & leg position \\
\hline$x_{1}$ & $\theta_{1} \cdot r_{1}$, Motor position in cable length \\
\hline$x_{2}$ & $\theta_{2} \cdot r_{2}$, Leg position in cable length \\
\hline$J_{1}$ & motor inertia \\
\hline$J_{2}$ & leg inertia \\
\hline$x_{3}$ & pretension \\
\hline$\Delta x$ & linear leg deflection, $\theta_{2} r_{2}-\theta_{1} r_{1}$ \\
\hline$z$ & deflection of the cable after the pulley function: \\
\hline & $\left(x_{3}+\Delta x\right)$ or $\left(x_{3}-\Delta x\right)$ \\
\hline$F_{\text {eff }}\left(x_{3}, \Delta x\right)$ & knee force, $\tau_{e f f} / r_{2}$ \\
\hline$G(z)$ & spiral pulleys; spring position as a function of $z$ \\
\hline$y$ & deflection of the spring, before the pulleys \\
\hline$F_{y}(y)$ & force function of the spring \\
\hline$F_{z}(z)$ & force on the cable after the pulley function \\
\hline
\end{tabular}

Table 1: Definitions of symbols describing the AMASC. 


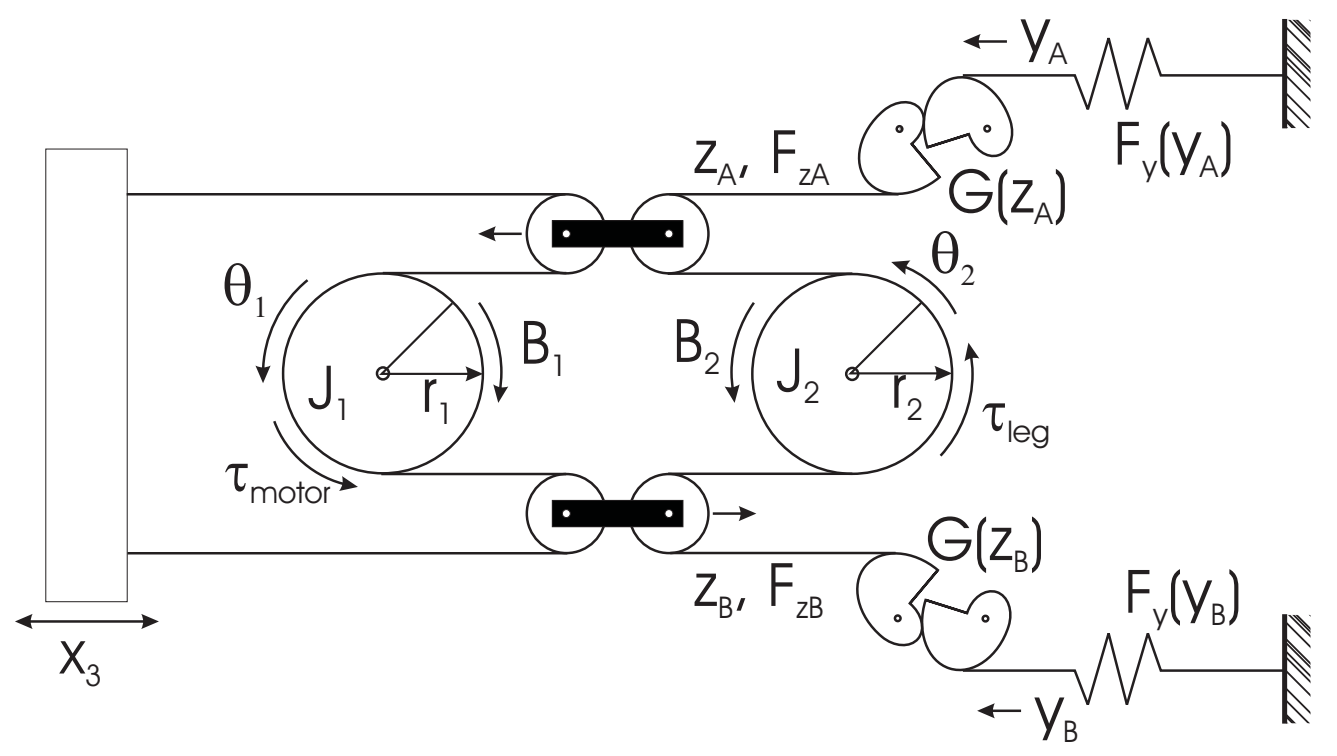

Figure 5: Cable routing diagram of the AMASC. $J_{1}$ and $J_{2}$ are pinned in place but can rotate freely; the spiral pulleys are also pinned in place but free to rotate. The remaining four pulleys are floating, and can move sideways as well as rotate. Refer to Table 1 for notation descriptions.

In order to create a low-friction, zero-backlash system, the AMASC utilizes a high-speed cable drive [31]. There is some stretch in the cable transmission, which adds series compliance to the system, and is incorporated into the effective spring constant of our model. The cables may flex in two planes and can easily be routed around joints, allowing the motors to be located remotely. They are not constrained to a single degree of freedom, like standard belts or gears. Figure 5 shows the cable routing, illustrating the role of each motor in the tension of the two springs. Also shown is the fact that a displacement of the leg $\left(\theta_{2}\right.$ or $\left.x_{2}\right)$ results in displacement of the motor $\left(\theta_{1}\right.$ or $\left.x_{1}\right)$, displacement of the springs, or some combination of the two. There is a speed reduction between the first and second pulleys not shown on the diagram; it is implemented using a combination of a block-and-tackle pulley mechanism and a difference in radii between $r_{1}$ and $r_{2}$. The speed reduction is physically located near the knee joint, but diagrammatically located near the motor, $\theta_{1}$. In all of our representations, the speed reduction is shown solely as a difference between $r_{1}$ and $r_{2}$. All friction related to the speed reduction is applied to $\theta_{1}$ and corresponds to $B_{1}$. The inertia of the speed reduction is added to the inertia of the motor, and corresponds to $J_{1}$.

A speed reducer amplifies the motor inertia by the square of the speed reduction; this amplification appears in the relatively large values of $M_{1}$. The transmission between $\theta_{2}$ and the springs has very low friction, and no speed reduction. Because the high-frequency behavior of the system is generally handled by the springs, low friction and inertia are most important in this part of the AMASC. The low-frequency behaviors of the system are handled by the motor, and thus friction and inertia can be overcome by relatively low-bandwidth software compensation.

Perhaps the most important aspect of the AMASC is the physically variable series compliance. As stated in Section 2, physical compliance is crucial for a running gait, while varying the compliance is a useful control strategy. Our physical compliance resides in unidirectional fiberglass plates, which have a relatively high work capacity on the order of $1000 \mathrm{~J} / \mathrm{kg}$. Varying the compliance of the AMASC is achieved in much the same way as in animals, with co-contraction of opposing nonlinear springs.

In the case of animals, the nonlinear spring is the muscle/tendon combination; in the case of the AMASC, the nonlinear spring is formed by a fiberglass plate in series with a set of spiral pulleys. The reduction ratio of the pulleys varies proportionally with the fiberglass spring deflection, to create some output spring function, such as $F_{z}(z)=K z^{2}$. Placing two such spring functions in direct opposition results in a single effective spring force function, $F_{\text {eff }}$. The resulting effective spring force is calculated by substituting $\left(x_{3}+\Delta x\right)$ and $\left(x_{3}-\Delta x\right)$ for $z$, where $x_{3}$ represents the pretension on the two nonlinear springs and $\Delta x$ represents the deflection from their rest position $\left(x_{2}-x_{1}\right)$. Combining the two forces results in

$$
F_{\text {eff }}\left(x_{3}, \Delta x\right)=F_{z}\left(x_{3}+\Delta x\right)-F_{z}\left(x_{3}-\Delta x\right) \text {. }
$$




\begin{tabular}{|r|c|l|}
\hline variable & value & description \\
\hline \hline$C$ & $0.1 m$ & center distance between axles \\
\hline$r$ & $0.05 m$ & radius of the round pulley \\
\hline$k$ & 0.2446489 & affects the "steepness" of the spiral \\
\hline$A$ & 0.017696 & scales the overall size of the spiral \\
\hline$\phi_{0}$ & -10.2078 & position of pulley when $z=0$ \\
\hline
\end{tabular}

Table 2: Measurements of the logarithmic spiral pulleys.

For the example quadratic springs, $F_{z}(z)=K z^{2}$ :

$$
\begin{aligned}
F_{\text {eff }}= & K\left(x_{3}+\left(x_{2}-x_{1}\right)\right)^{2}-K\left(x_{3}-\left(x_{2}-x_{1}\right)\right)^{2} \\
F_{\text {eff }}= & K\left(x_{3}^{2}-2 x_{3} x_{1}+2 x_{3} x_{2}+x_{1}^{2}-2 x_{1} x_{2}+x_{2}^{2}\right)- \\
& K\left(x_{3}^{2}+2 x_{3} x_{1}-2 x_{3} x_{2}+x_{1}^{2}-2 x_{1} x_{2}+x_{2}^{2}\right) \\
F_{\text {eff }}= & 4 K x_{3}\left(x_{2}-x_{1}\right)
\end{aligned}
$$

In this manner, the stiffness of the resulting system can be changed by adjusting the pretension, $x_{3}$. Notice that the pretension affects the force as much as the displacement $\left(x_{2}-x_{1}\right)$. The effective spring force $F_{\text {eff }}$ is linear with respect to displacement (in this specific case), but its stiffness is now adjustable. In practice, the rate at which this parameter can be varied depends on the actuator and transmission used. Our prototype is intended for relatively slow changes at low force, such as during the flight phase of a running gait.

The pulley function, $G(z)$, is a design freedom and can be changed to impart a nearly arbitrary function to the spring/pulley system, $F_{z}(z)$. Logarithmic spiral pulleys were initially chosen because the spring function of the bending fiberglass plates was unknown, because the desired spring function was unknown, and because two logarithmic spirals mesh correctly and provide a stiffening function [32]. Our logarithmic spiral pulleys are described by the following equations:

$$
\begin{aligned}
& R_{1}=A e^{k \phi} \\
& R_{2}=C-A e^{k \phi}
\end{aligned}
$$

Where $\phi$ is the angle of rotation of the pulley,

$$
\phi(z)=\frac{z}{r}
$$

These pulleys exhibit the velocity transfer function

$$
\frac{d y}{d z}=\frac{d G}{d z}(z)=\frac{A e^{k \frac{z}{r}}}{C-A e^{k \frac{z}{r}}}
$$

and exhibit the position function

$$
y=G(z)=r\left(\phi_{0}-\frac{1}{k} \ln \left(C-A e^{k \frac{z}{r}}\right)\right) .
$$

The choice of a logarithmic spiral was somewhat arbitrary. Before new pulleys can be designed, however, two functions must be considered: the desired output force function, $F_{z}(z)$, and the measured fiberglass spring force function $F_{y}(y)$. The desired spring force function $F_{z}(z)$ can be described in terms of the pulley transmission function $y=G(z)$, where $z$ is the extension of the cable out of the pulley. We calculate $F_{z}(z)$ by computing the virtual work:

$$
\begin{aligned}
F_{z}(z) d z & =F_{y}(y) d y \\
y=G(z), & d y=\frac{d G}{d z}(z) d z \\
F_{z}(z) d z & =F_{y}(G(z)) \frac{d G}{d z}(z) d z \\
F_{z}(z) & =F_{y}(G(z)) \frac{d G}{d z}(z) .
\end{aligned}
$$




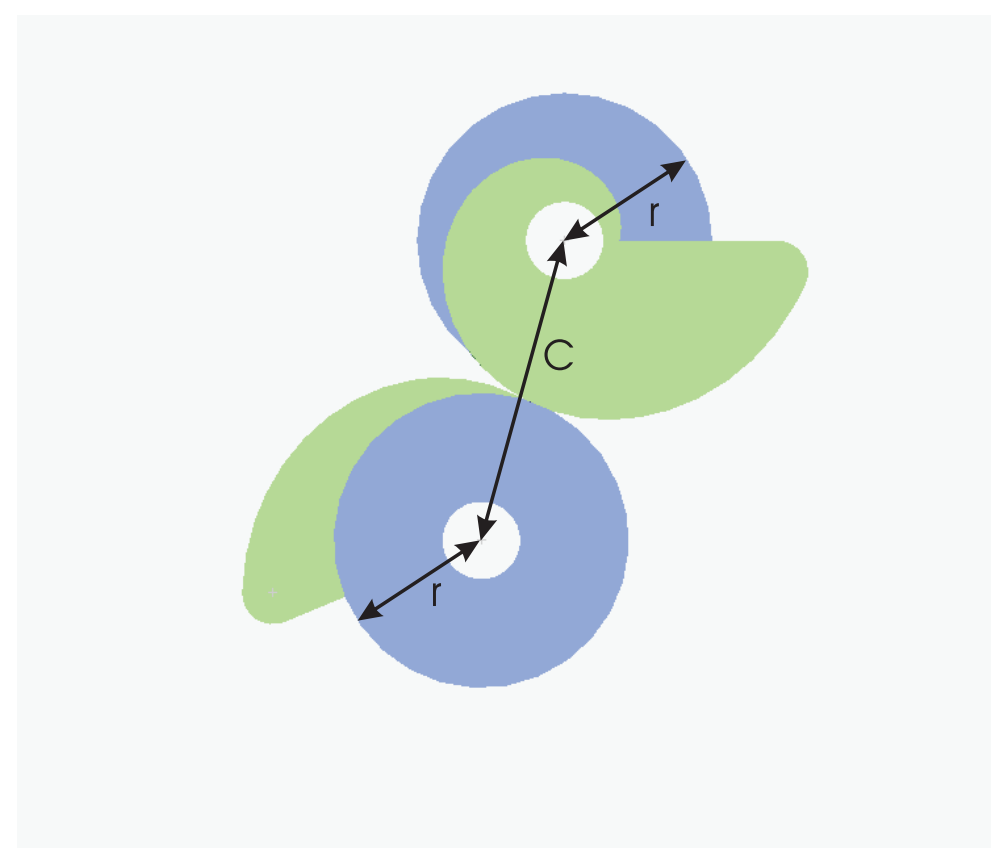

Figure 6: The logarithmic spiral pulleys used on the prototype AMASC

Given the desired $F_{z}$ and the spring function $F_{y}$, we can solve Equation 5 for the pulley function $G(z)$. With this pulley function, the relationship between angular velocities of two members, $\frac{d G}{d z}(z)$, can be determined. Given the center distance $C$ between the pulleys, the polar equations describing the spiral shape of both pulleys are:

$$
\begin{aligned}
& R_{1}=\frac{C \frac{d G}{d z}(z)}{1+\frac{d G}{d z}(z)} \\
& R_{2}=C-R_{1} .
\end{aligned}
$$

Even if the fiberglass springs have some unusual stiffness function $F_{y}(y)$, the spiral pulley function $G_{z}(z)$ can account for it, and can create any odd output function $F_{\text {eff }}$. The following derivation shows that $F_{\text {eff }}$ must be an odd function for any arbitrary $F_{z}$.

Let $F_{z}(z)$ described by a Taylor series:

$$
F_{z}(z)=a_{0}+a_{1} z+a_{2} z^{2}+a_{3} z^{3} \ldots
$$

We can substitute $x_{3}+\Delta x$ in for $z$

$$
F_{z}\left(x_{3}+\Delta x\right)=a_{0}+a_{1}\left(x_{3}+\Delta x\right)+a_{2}\left(x_{3}+\Delta x\right)^{2}+a_{3}\left(x_{3}+\Delta x\right)^{3}
$$

This will give us a polynomial function in terms of $x_{3}$ and $\Delta x$ :

$$
F_{z}\left(x_{3}+\Delta x\right)=F^{\prime}\left(x_{3}, \Delta x\right)
$$

We can break this function, $F^{\prime}$, into the even and odd powers of $\Delta x, F_{\text {even }}^{\prime}$ and $F_{\text {odd }}^{\prime}$ :

$$
F^{\prime}\left(x_{3}, \Delta x\right)=F_{\text {even }}^{\prime}\left(x_{3}, \Delta x\right)+F_{\text {odd }}^{\prime}\left(x_{3}, \Delta x\right)
$$

Therefore, the spring force at the knee becomes

$$
F_{\text {eff }}=\left(F_{\text {even }}^{\prime}\left(x_{3}, \Delta x\right)+F_{\text {odd }}^{\prime}\left(x_{3}, \Delta x\right)\right)-\left(F_{\text {even }}^{\prime}\left(x_{3},-\Delta x\right)+F_{\text {odd }}^{\prime}\left(x_{3},-\Delta x\right)\right) .
$$




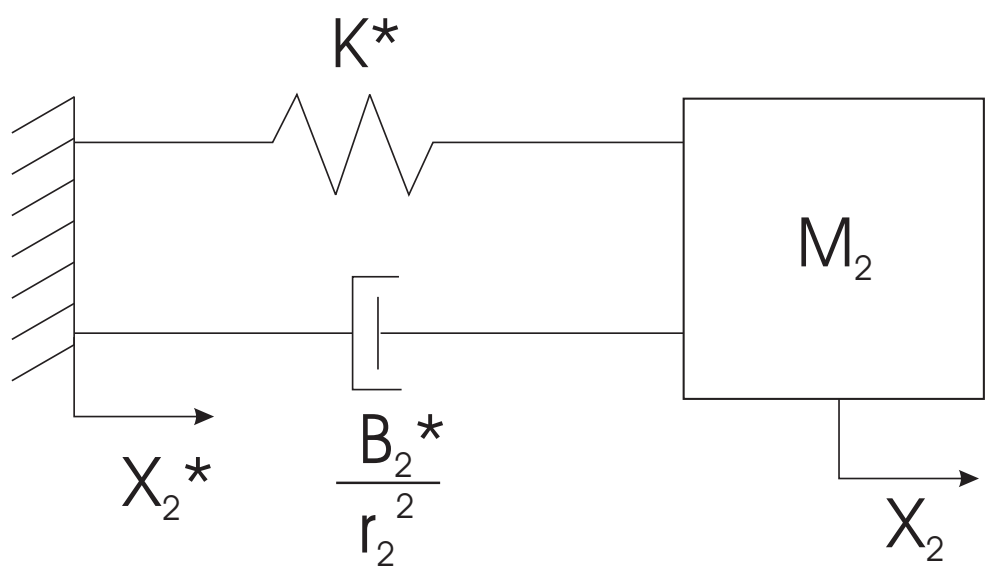

Figure 7: The ideal case of the AMASC, with three parameters: spring rest length, $x_{2}^{*}$, spring stiffness, $K^{*}$, and knee joint damping, $B^{*}$.

And because

$$
\begin{aligned}
& F_{\text {even }}^{\prime}\left(x_{3},-\Delta x\right)=F_{\text {even }}^{\prime}\left(x_{3}, \Delta x\right) \\
& F_{\text {odd }}^{\prime}\left(x_{3},-\Delta x\right)=-F_{\text {odd }}^{\prime}\left(x_{3}, \Delta x\right),
\end{aligned}
$$

we can substitute for $F_{z}$ in Equation 1 and obtain the result:

$$
\begin{gathered}
F_{\text {eff }}=F_{\text {even }}^{\prime}\left(x_{3}, \Delta x\right)+F_{\text {odd }}^{\prime}\left(x_{3}, \Delta x\right)-F_{\text {even }}^{\prime}\left(x_{3}, \Delta x\right)+F_{\text {odd }}^{\prime}\left(x_{3}, \Delta x\right) \\
F_{\text {eff }}=2 F_{\text {odd }}^{\prime}\left(x_{3}, \Delta x\right)
\end{gathered}
$$

The fact that $F_{\text {eff }}$ is necessarily odd indicates that the spring function is rotationally symmetric about the origin. In other words, when deflecting the joint in one direction, the resulting force will be exactly opposite to the force that results from deflecting the joint the same amount in the other direction. This result may not be interesting in the context of legged locomotion, since most locomotion tasks will apply force in one direction only.

\subsection{Control System Design}

The control system is designed for the mechanical model shown in Figure 4, and is intended to accomplish two basic tasks. The first task is to adjust the mechanism configuration so its physical properties match the commanded spring stiffness. This adjustment is accomplished with a PID position controller and a spring-cancellation feed-forward torque on the pretension motor. Because the specific position of this motor, $x_{3}$, corresponds to a specific effective stiffness $K_{\text {eff }}\left(x_{3}\right)$, no further control is currently required.

The second task is to actively control the motor position, $x_{1}$, so that the force applied by the leg spring, $F_{z}(z)$, matches the spring force that would be created by the ideal, correctly tuned system. This ideally tuned system is shown in Figure 7, with desired leg stiffness, $K^{*}$, and desired set point $x_{2}^{*}$. When the physical stiffness $K_{\text {eff }}$ matches the desired stiffness $K^{*}$, then the motor position $x_{1}$ will be commanded to match the ideal system's desired set point $x_{2}^{*}$. When $K_{\text {eff }}$ does not match $K^{*}$, then $x_{1}$ must move in some additional corrective trajectory.

The desired motor position $x_{1}$ is calculated by setting the force of the actual springs to match the spring force of the ideal system:

$$
F_{\text {eff }}\left(\Delta x, x_{3}\right) r_{2}+\frac{B_{2}}{r_{2}} \dot{x}_{2}=\left(x_{2}-x_{2}^{*}\right) K^{*} r_{2}+\frac{B_{2}^{*}}{r_{2}} \dot{x}_{2}
$$

Assuming the dependence of $F_{\text {eff }}$ on $\Delta x$ is linear $\left(F_{\text {eff }}=K_{\text {eff }}\left(x_{3}\right) \Delta x\right)$, we can solve this equation for $x_{1}$ to calculate the desired position, $x_{1}^{*}$, and its derivative, $\dot{x}_{1}$ 


$$
\begin{aligned}
x_{1}^{*}= & \frac{K^{*}}{K_{e f f}}\left(x_{2}^{*}-x_{2}\right)-\frac{B_{2}^{*}-B_{2}}{K_{e f f} r_{2}^{2}} \dot{x}_{2}+x_{2} \\
\dot{x}_{1}^{*}= & -\frac{K^{*}}{K_{e f f}} \dot{x}_{2}-\frac{K^{*}}{K_{e f f}^{2}} \dot{K}_{e f f}\left(x_{2}^{*}-x_{2}\right)-\frac{B^{*}-B_{2}}{K_{e f f} r_{2}^{2}} \ddot{x}_{2} \\
& +\frac{B^{*}-B_{2}}{K_{e f f}^{2} r_{2}^{2}} \dot{K}_{\text {eff }} \dot{x}_{2}+\dot{x}_{2}
\end{aligned}
$$

Note that when the mechanism matches the desired system (i.e. $K^{*}=K_{\text {eff }}$ and $B^{*}=B_{2}$ ), the above equations reduce to $x_{1}^{*}=x_{2}^{*}$ and $\dot{x}_{1}^{*}=0$.

We then apply a PD controller on $x_{1}$ to move it to the desired position, along with a feed-forward spring cancellation force to hold it against the force applied by the springs:

$$
F_{\text {com }}=K_{P}\left(x_{1}^{*}-x_{1}\right)+K_{D}\left(\dot{x}_{1}^{*}-\dot{x}_{1}\right)-F_{\text {eff }}\left(x_{3}, \Delta x\right) .
$$

With the spring cancellation force, the PD control can adjust $x_{1}$ as if it were an independent mass, without the attached spring and associated dynamics. There are two limitations in this approach that introduce error. First, because $F_{\text {eff }}$ is a function composed of the logarithmic spiral pulleys and the unknown fiberglass spring function, it is necessarily an approximation. We used a linear approximation of the knee spring, $F_{\text {eff }}=K_{\text {eff }}\left(x_{3}\right) \Delta x$, introducing error between the calculated force and the applied force, most pronounced at the extremes of deflection and pretension. In addition, when simulating a spring stiffness that is outside the physical range of the AMASC, the calculated location $x_{1}^{*}$ will only be correct to the accuracy of the approximation.

The second source of error comes from the bandwidth limitation on $x_{1}$. When trying to simulate a stiffness at high frequency, the inertia of $M_{1}$ will limit the acceleration of $x_{1}$, and the system will revert to the behavior of its natural dynamics, instead of the desired behavior. However, because the stiffness of the AMASC is adjustable, this error can only happen when the desired stiffness is outside the range of the mechanism, or when the mechanism is in the process of adjusting to the correct stiffness.

\section{Simulation, Results and Comparison}

This section describes the methods and approaches used the characterize the physical properties of the AMASC and to build an accurate simulation. After illustrating the similarity of the simulation to the real actuator, we demonstrate a simulated running robot, and compare its performance using both ideal actuation, and our simulated AMASC.

\subsection{Static Compliance Characterization}

An accurate representation of the fiberglass springs and their interaction at the knee was required for correct simulation and control. To gather data on $F_{\text {eff }}$ (shown in Figure 8), we applied a series of spring set point and pretension values, and recorded the motor and leg positions and the force applied by the leg. We then applied several different curvefitting methods, to create a representation of the data in which applied force is a function of spring pretension and spring set point.

As can be seen in Figure 10, the AMASC spring function becomes stiffer at increasing levels of pretension. The spring function is not exactly linear in deflection, although this can be remedied through pulley design as discussed in section Section 3.1. It is also apparent in Figure 10 that there is some hysteresis due to mechanical friction. This friction increases with increasing tension (and higher bearing forces) in the system, as seen in Figure 11.

There are numerous methods that could be used to fit the available data, each with benefits and drawbacks. One of the most basic methods is a Taylor polynomial. Although the deflection curve is clearly not linear, it is a relatively flat curve; so we applied a fit that is linear in deflection and quadratic in pretension, resulting in a 3 term polynomial:

$$
F_{\text {eff }}\left(x_{3}, \Delta x\right)=a_{0} \Delta x+a_{1} x_{3} \Delta x+a_{2} x_{3}^{2} \Delta x
$$

This fit allows us to easily calculate the pretension position for a desired stiffness, and as it is linear in deflection, we can use the control equations described in Section 3.2, which assume a linear dependence of $F_{\text {eff }}$ on $\Delta x$. It provides 


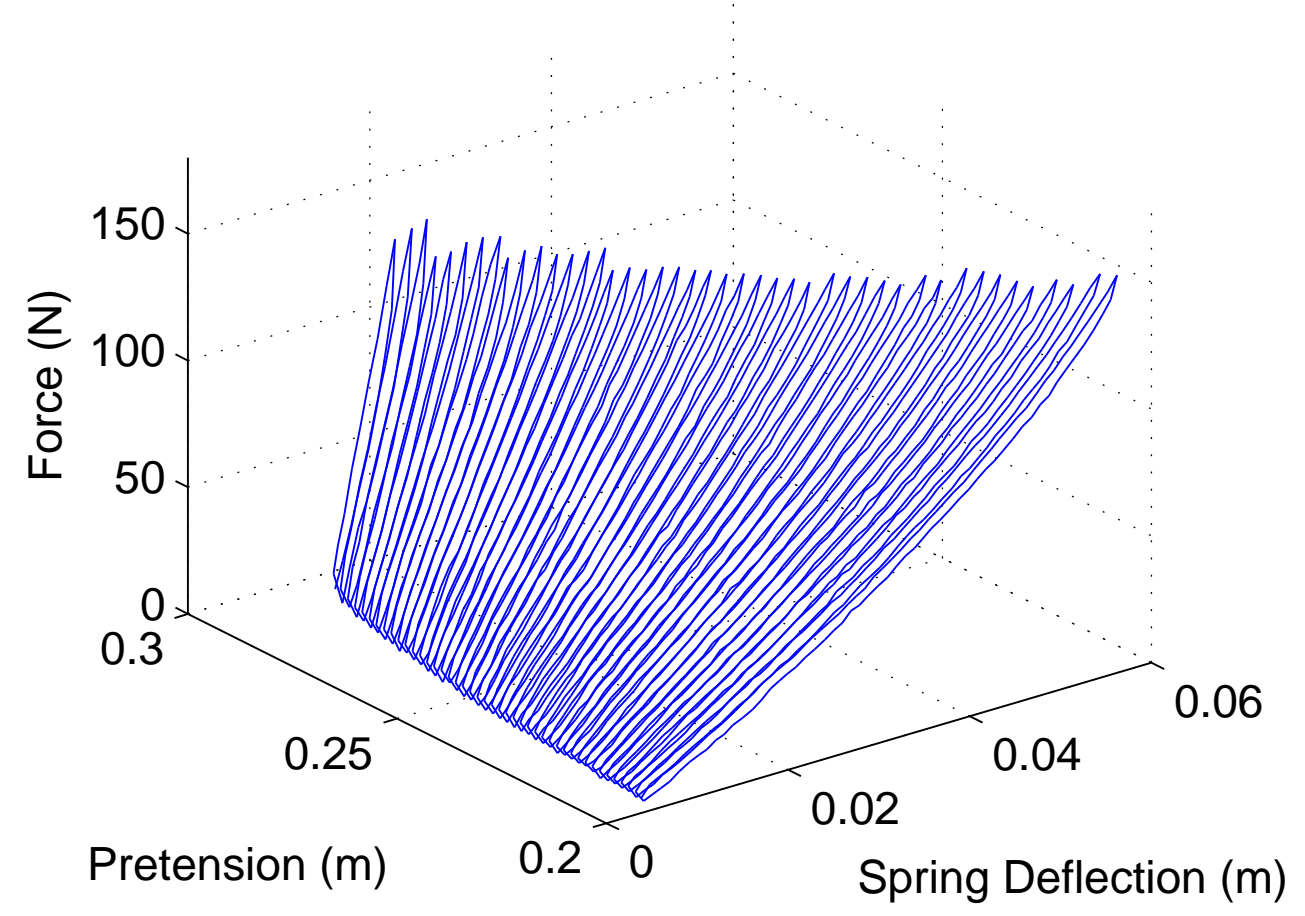

Figure 8: 3D plot of spring function data

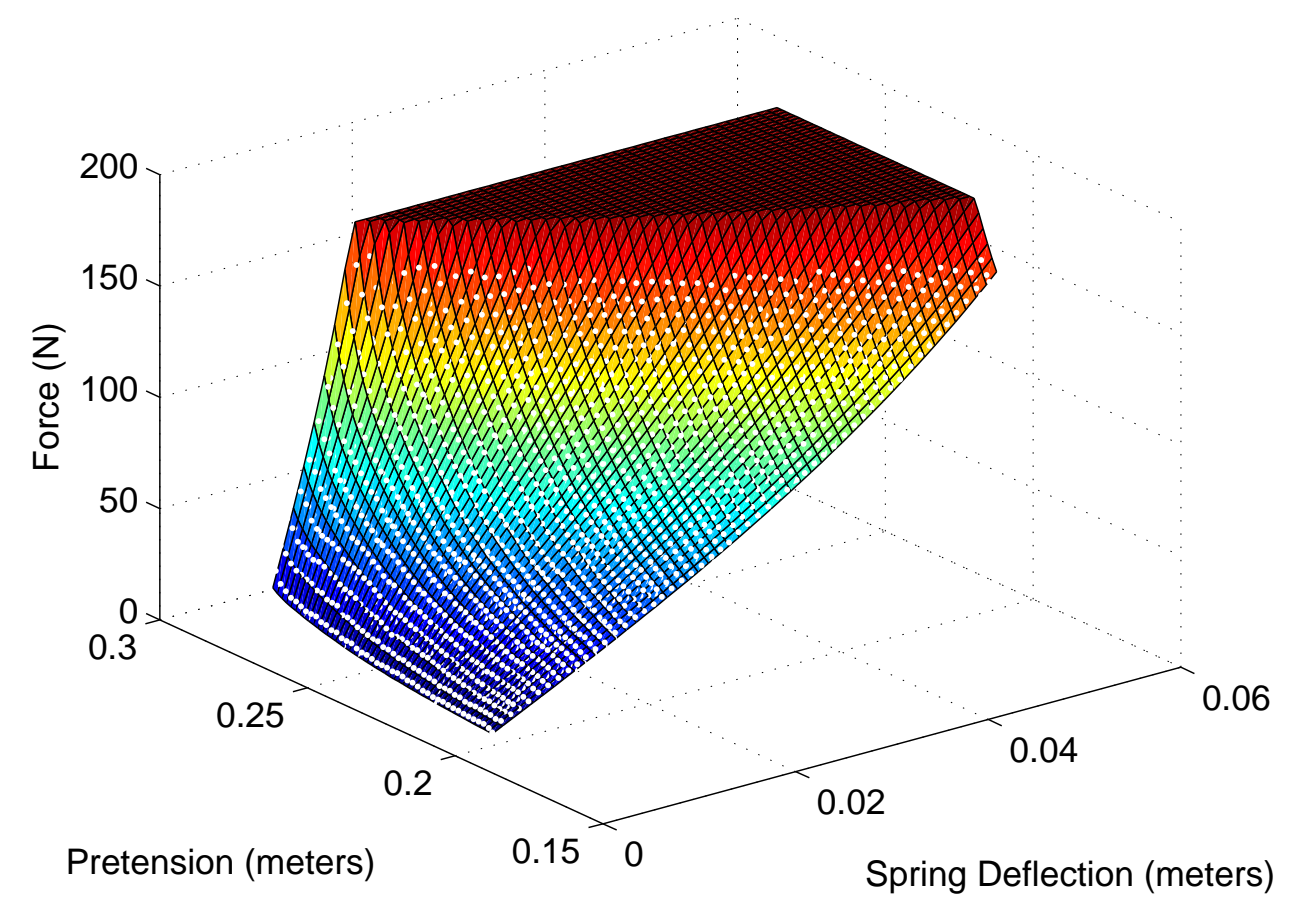

Figure 9: Surface plot of one spring function curve fit, with data points shown as white dots 


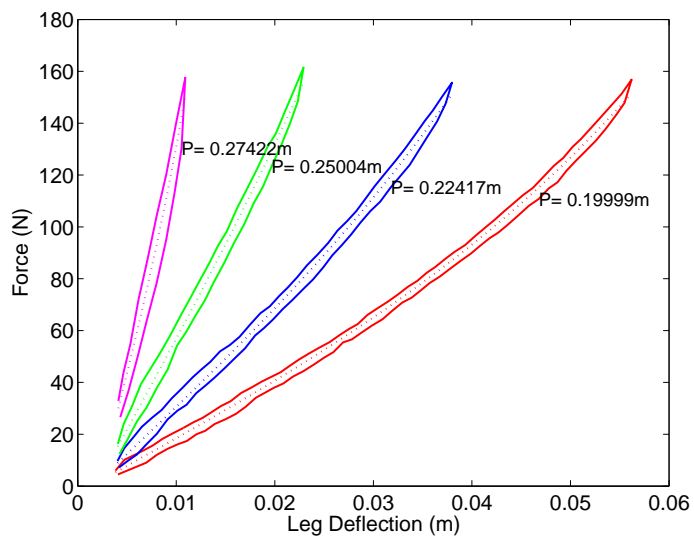

Figure 10: Spring force response at four different pretension settings, with one possible curve fit (dotted line)

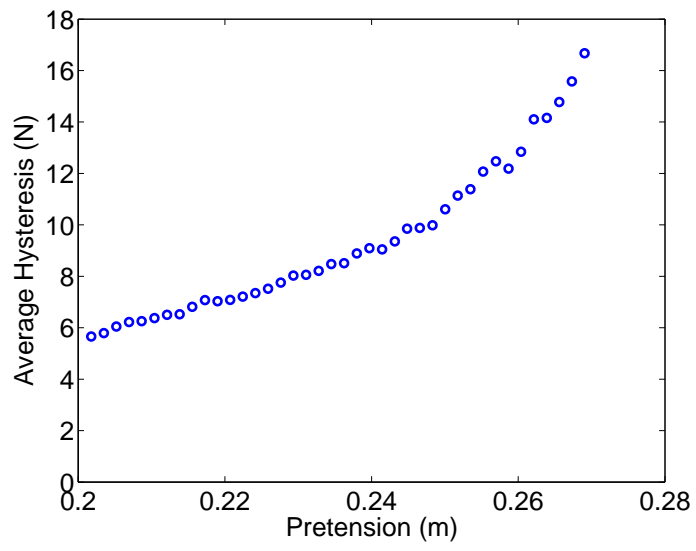

Figure 11: Hysteresis in the knee due to friction, shown as a function of pretension.

a reasonable fit of the data, although not as good as some other possible approaches. Figure 12(a) shows the absolute error between this approximation and the actual data.

A large (14-term) polynomial in both deflection and pretension yielded a much better fit than the 3-term polynomial, with lower error, as shown in Figure 12(b). Unfortunately, this function cannot be inverted in closed form to solve for $x_{3}$, and it is not linear in deflection for use in the control system equations.

One drawback to using the polynomial fit to characterize $K_{\text {eff }}$ is that we are ignoring the fact that the pulleys are a logarithmic spiral. This is a known function, and we are basically attempting to fit a polynomial to this known function, which will introduce a certain amount of unnecessary error. In addition, we would like to know the spring function of the physical springs, $F_{y}(y)$, before the force is modified by the logarithmic pulleys. Knowing the spring function of the physical springs will allow future pulley designs to compensate for undesirable properties of $F_{y}$ and create the desired spring function $F_{z}$. By mathematically separating the springs from the logarithmic spirals and then applying a curve fit solely to the physical springs, our error is reduced, the polynomial is simplified, and we find that the physical springs are very nearly linear.

The force data collected from the actuator measures knee stiffness, $F_{\text {eff }}$, which is created by the two opposing springs as described in Equation 1. The pulley functions are known, described in Equations 3 and 4.

Substituting $\frac{d G(z)}{d z}$ into the equation for $F_{z}$ (Equation 5) results in

$$
F_{z}(z)=\frac{A e^{k \frac{z}{r}}}{C-A e^{k \frac{z}{r}}} F_{y}(y) .
$$

If $F_{y}(y)$ is represented as a Taylor series,

$$
F_{z}(z)=\frac{A e^{k \frac{z}{r}}}{C-A e^{k \frac{z}{r}}}\left[a_{0}+a_{1} y+a_{2} y^{2}+a_{3} y^{3} \ldots\right]
$$

and $G(z)$ is substituted for $y$,

$$
\begin{aligned}
& F_{z}(z)= \\
& \quad \frac{A e^{k \frac{z}{r}}}{C-A e^{k \frac{z}{r}}}\left[a_{0}+a_{1} r\left(\phi_{0}-\frac{1}{k} \ln \left(C-A e^{k \frac{z}{r}}\right)\right)+a_{2}\left(r\left(\phi_{0}-\frac{1}{k} \ln \left(C-A e^{k \frac{z}{r}}\right)\right)\right)^{2}\right. \\
& \left.\quad+a_{3}\left(r\left(\phi_{0}-\frac{1}{k} \ln \left(C-A e^{k \frac{z}{r}}\right)\right)\right)^{3} \cdots\right]
\end{aligned}
$$

We now have an equation for $F_{z}$ in terms of a polynomial fit of $F_{y}$. We can substitute this result into Equation 1 to acquire an equation for $F_{\text {eff }}$. To simplify this equation, we construct the following definitions:

$$
H=\frac{A e^{k \frac{x_{3}+\Delta x}{r_{1}}}}{C-A e^{k \frac{x_{3}+\Delta x}{r_{1}}}}
$$




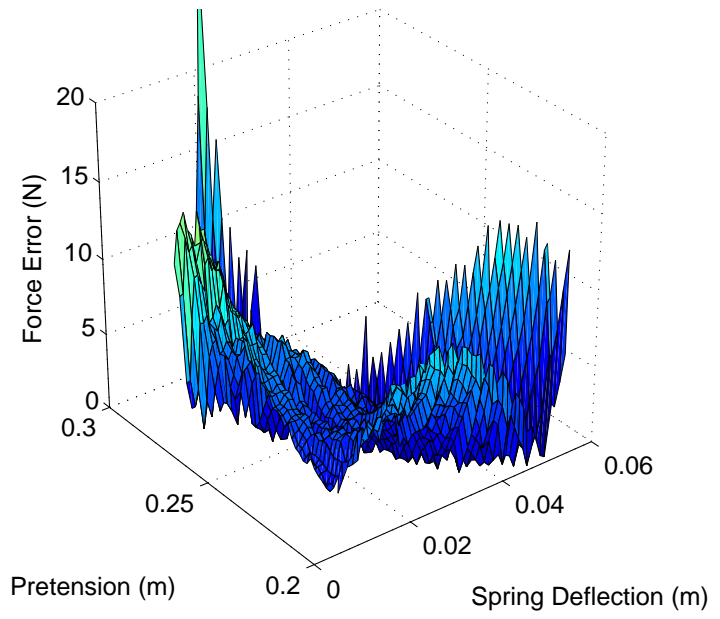

(a) 3-term polynomial, linear in deflection

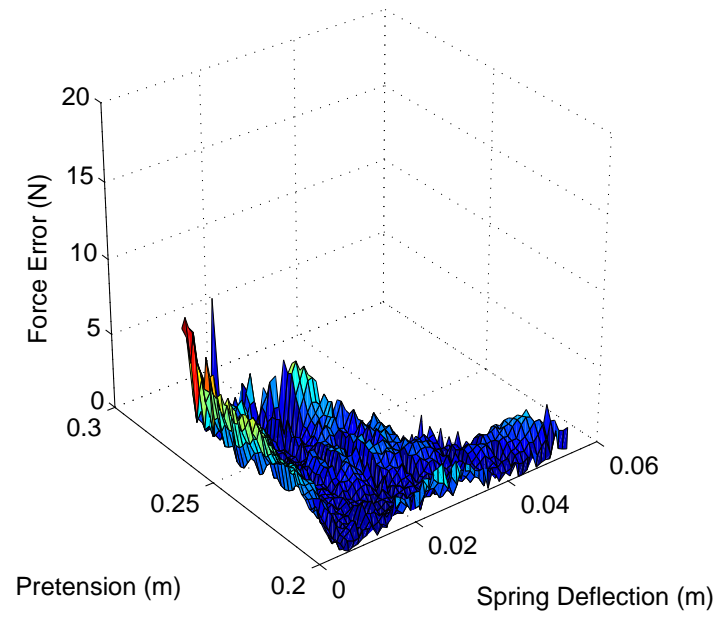

(b) Many-term polynomial

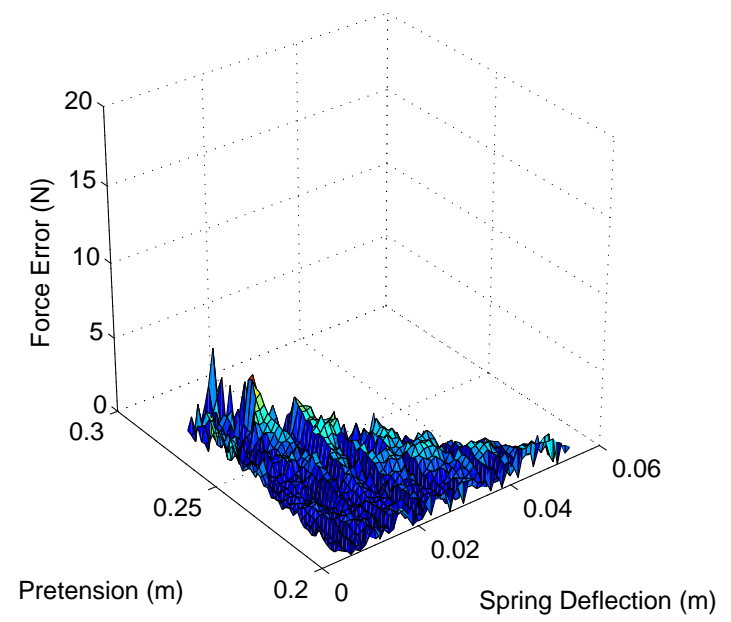

(c) linear $F_{y}(y)$ passed through pulleys

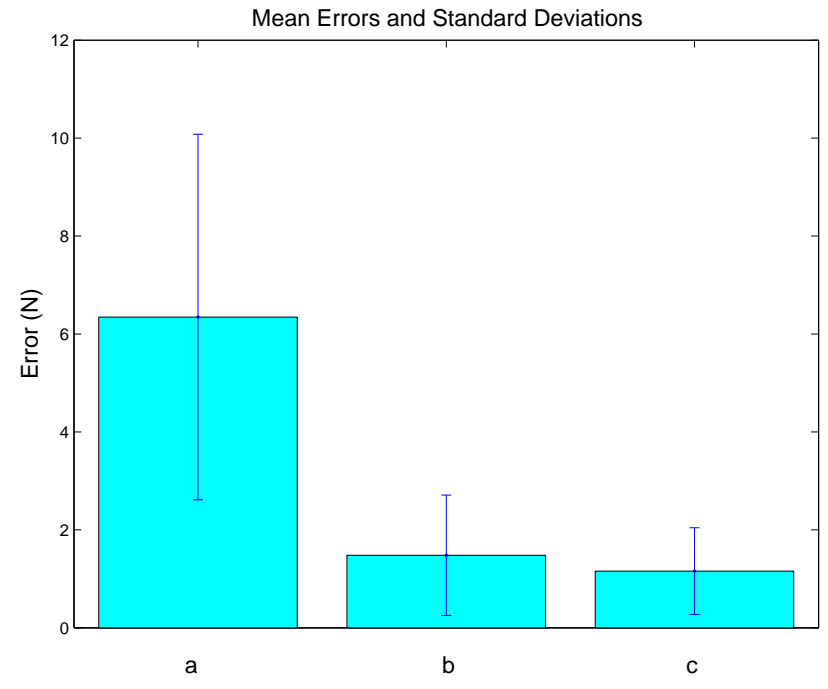

(d) Mean Error for data fits

Figure 12: Plots of absolute error between data points and curve fits 


$$
\begin{aligned}
I & =r_{1}\left(\phi_{0}-\frac{1}{k} \ln \left(C-A e^{k \frac{x_{3}+\Delta x}{r_{1}}}\right)\right) \\
J & =\frac{A e^{k \frac{x_{3}-\Delta x}{r_{2}}}}{C-A e^{k \frac{x_{3}-\Delta x}{r_{2}}}} \\
L & =r_{2}\left(\phi_{0}-\frac{1}{k} \ln \left(C-A e^{k \frac{x_{3}-\Delta x}{r_{2}}}\right)\right)
\end{aligned}
$$

By substituting these variables and Equation 10 into Equation 1, we arrive at the equation for $F_{\text {eff }}$,

$$
F_{\text {eff }}\left(x_{3}, \Delta x\right)=H\left(a_{0}+a_{1} I+a_{2} I^{2}+a_{3} I^{3}+\ldots\right)-J\left(a_{0}+a_{1} L+a_{2} L^{2}+a_{3} L^{3}+\ldots\right),
$$

which can be factored into

$$
F_{\text {eff }}\left(x_{3}, \Delta x\right)=a_{0}(H-J)+a_{1}(H I-J L)+a_{2}\left(H I^{2}-J L^{2}\right)+\ldots
$$

This form is very useful for fitting a curve to the data. It allows us to calculate $a_{0} \ldots a_{n}$, which correspond to the physical spring force polynomial before it is passed through the pulleys. We found that using a simple linear fit of the physical spring resulted in a good approximation, as shown in Figure 12(c). This fit was used to generate the surface in Figure 9 and the dotted curves in Figure 10. Not only is this curve fitting method more accurate than any of the polynomial fits, it also has the advantage that it can be used to calculate spring cancellation forces for both the control on $x_{1}$ and $x_{3}$. A standard polynomial curve fit of $F_{\text {eff }}$ can only calculate a spring cancellation force for $x_{1}$. In addition, because this method describes how the physical spring (before the pulley function) behaves, pulleys can be designed to provide the desired $F_{z}(z)$. Unfortunately, this fit cannot be easily inverted to solve for the desired positions of $x_{1}$ or $x_{3}$. In our controller, the linear polynomial curve fit is used to calculate the desired values of $x_{1}$ and $x_{3}$, while Equation 11 is used for spring cancellation forces and other calculations that do not require inversion.

\subsection{Dynamic Actuator Simulation}

Dynamic simulation of the AMASC mechanism was done using SD/FAST ${ }^{1}$, based on the model shown in Figure 4(a). The springs are approximated as linear springs passed through the logarithmic spiral pulleys (i.e. the fit shown in Figure 12(c) and described by Equation 11). The dynamics of the pretension motor are conservatively approximated by a constant-velocity trajectory to the desired setting. Physical parameters such as link length and inertia were measured from the SolidWorks model and from the AMASC prototype. The values are these parameters are given in Table 3.

\begin{tabular}{|r|c|c|l|}
\hline variable & value & variable & value \\
\hline \hline$J_{1}$ & $0.00134 \mathrm{~kg} \cdot \mathrm{m}^{2}$ & $M_{1}=\frac{J_{2}}{r_{2}^{2}}$ & $59.6 \mathrm{~kg}$ \\
\hline$J_{2}$ & $0.085 \mathrm{~kg} \cdot \mathrm{m}^{2}$ & $M_{2}=\frac{J_{1}}{r_{1}^{2}}$ & $8.5 \mathrm{~kg}$ \\
\hline$B_{1}$ & $0.0517 \mathrm{~N} \cdot \mathrm{m} \cdot \mathrm{s} / \mathrm{rad}$ & $r_{1}$ & $0.00474 \mathrm{~m}$ \\
\hline$B_{2}$ & $0.38 \mathrm{~N} \cdot \mathrm{m} \cdot \mathrm{s} / \mathrm{rad}$ & $r_{2}$ & $0.1 \mathrm{~m}$ \\
\hline
\end{tabular}

Table 3: Physical properties used for simulation

The prototype AMASC was initially compared to its simulation by applying a step position input to the set-point of both systems. Figure 13(a) shows that the responses are very similar. One difference is that the oscillations of the prototype AMASC are damped once the amplitude of the oscillations becomes small. We hypothesize that this is due to frictional effects that are not accurately modeled by the viscous damping included in the model.

For a more comprehensive comparison, the prototype and simulated AMASC were tested across a range of frequencies and stiffness values. Figure 13(b) shows the similar responses of the prototype and simulation as the motor is commanded a sine wave position function through a range of frequencies at a series of ascending pretension values.

${ }^{1}$ SD/FAST is a trademark of Symbolic Dynamics, Inc. and Parametric Technology Corporation 

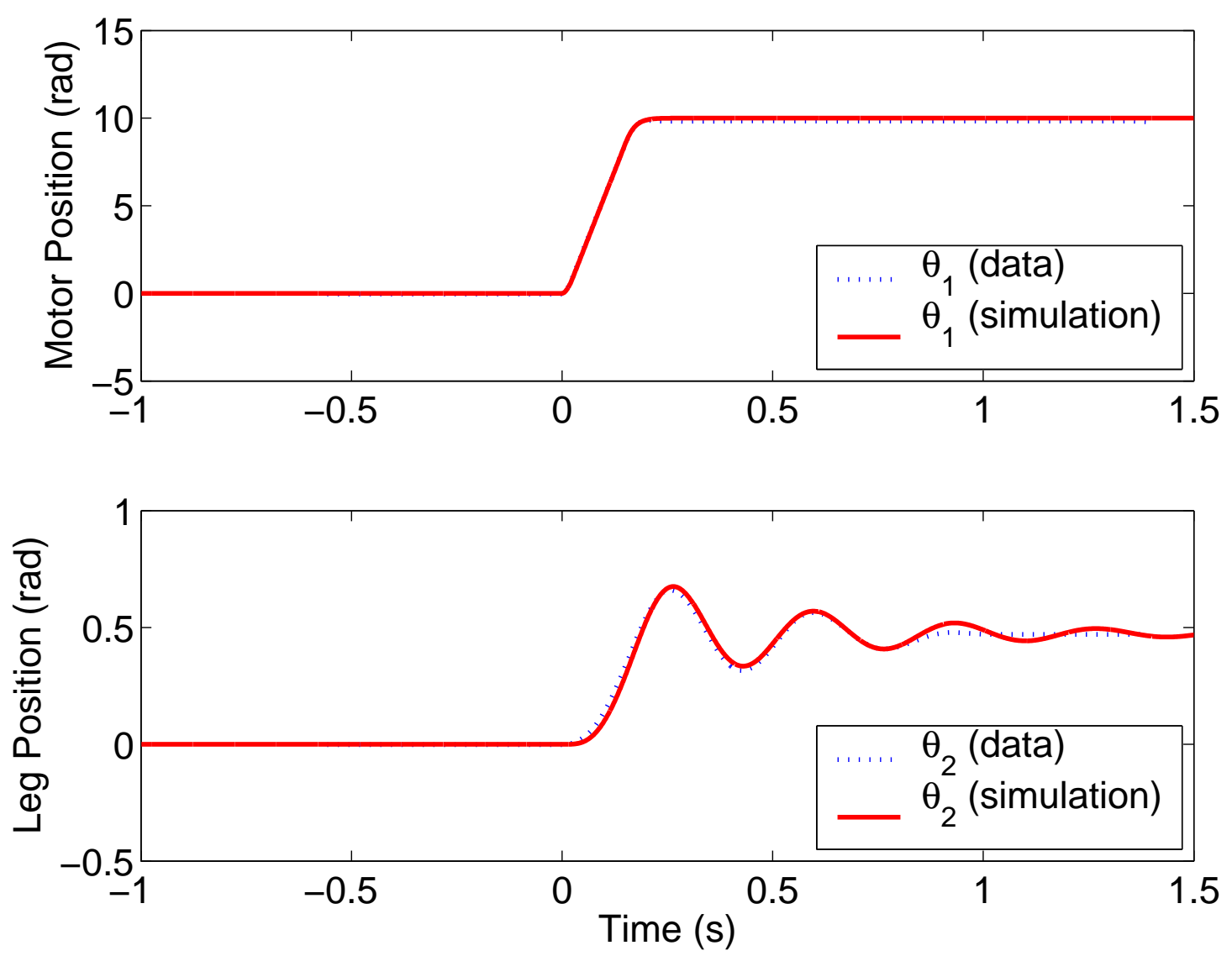

(a) Motor and leg response to a step input

Frequency plots of physical and simulated actuator

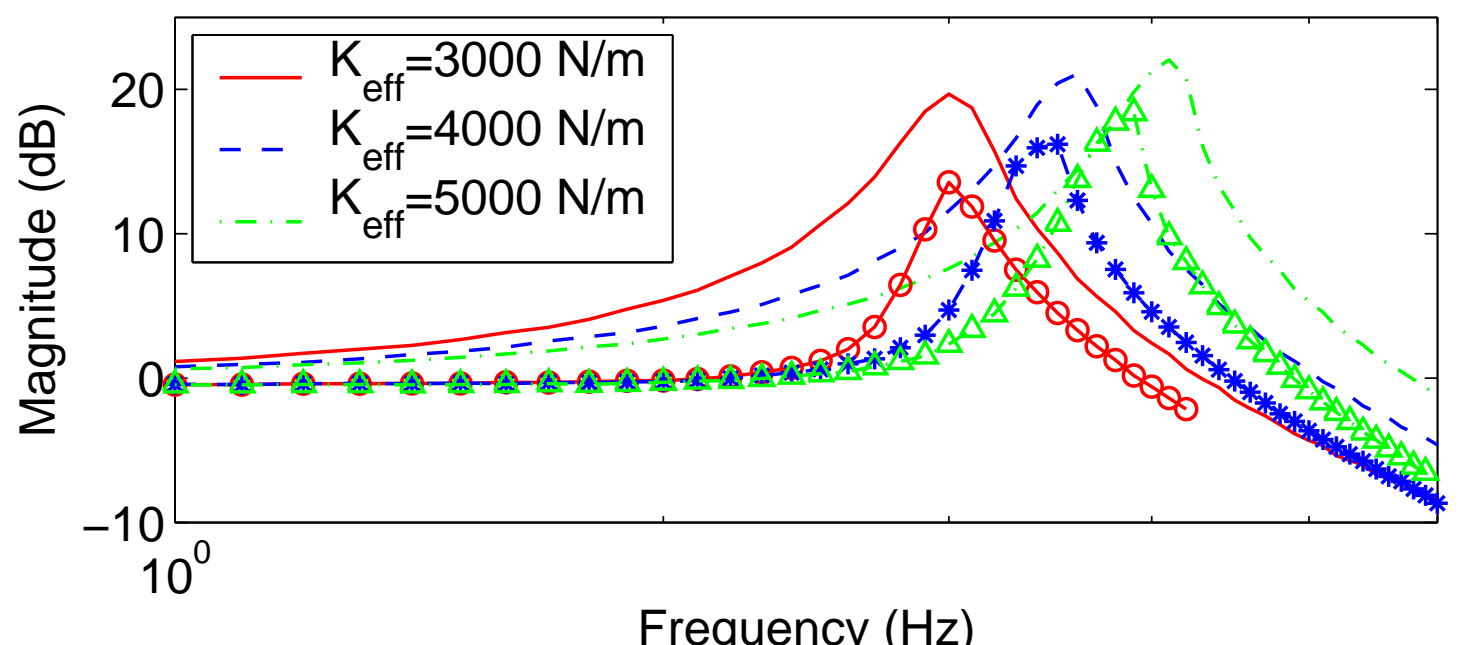

(b) Ratio of leg position to motor position, showing similar behavior at a range of frequencies and stiffnesses.

Figure 13: Comparison of prototype AMASC and simulation AMASC 
We hypothesize again that differences in the simulation and prototype are mostly caused by unmodeled frictional effects, such as stiction on the fiberglass springs. Part of the error may also be caused by our spring fit; it has some error, and is not an exact representation of the physical system.

These tests do not demonstrate the performance of the AMASC system, they merely show that the physical AMASC and simulated AMASC behave similarly at a range of frequencies. To measure the performance of any actuator, experiments should test how well it demonstrates desired dynamics under various conditions. For the specific case of SLIP model running, the AMASC should behave like a spring with a certain stiffness. In order to deal with frequent ground impacts during a running gait, the AMASC should exhibit this behavior to an arbitrarily high frequency, which is not possible for a standard gearmotor system.

A Bode test demonstrated the bandwidth limitations of the simulated AMASC. The input to the Bode plot was the leg position, which followed a sine wave. The output of the system is spring force. The three different Bode plots shown in Figure 14 represent three different commanded stiffness values $K^{*}$, with a constant physical stiffness, $K_{\text {eff }}$. These plots show the frequency limit of the AMASC when attempting to simulate a system softer or stiffer than its mechanical adjustment, and also demonstrate the lack of a bandwidth limit when the AMASC is tuned properly.

\subsection{Running Simulation}

We implemented a simulated running robot in SD/FAST based on the SLIP model. Raibert-style controllers were implemented as described in "Legged Robots that Balance"[33], with slight modifications. Raibert-style hopping height controllers insert a fixed amount of energy by changing the set-point during stance, causing the hopping height to converge to some value. Our modification calculates exactly how much energy must be added for the desired hopping height and speed, and adjusts the set-point to add the appropriate amount of energy.

The running simulation was first created with an ideal actuator placed at each joint, such that we could apply any stiffness and set-point instantly. We then converted the actuator in this running simulation to the simulation of the AMASC (see Figure 15) as described in Section 4.2. Because the prototype AMASC currently has very soft springs for bench testing, we adjusted the simulation so that the simulated fiberglass springs were ten times stiffer than the prototype's springs, which is more appropriate for a running gait. With the stiffer simulated fiberglass springs, we could reach the commanded stiffness without being outside the range of the experimental curve fits.

The simulated robot continued running with the AMASC, with no changes to the running controller from the simulation with the ideal knee spring. The resulting difference in the behavior of the running robot is shown in Figure 16. Note that the hopping height and stride length decrease slightly. This change is due to the fact that the set-point of the AMASC cannot change instantly, and thus slightly less energy is inserted into the running gait than with an ideal knee spring.

\section{Discussion}

\subsection{SEA comparison}

Although the AMASC is designed to match the desired natural dynamics for a particular SLIP-model running gait, it can be operated in the same manner as the MIT Series Elastic Actuator by disabling the variable stiffness and implementing an appropriate software controller. With this modification, the AMASC has the exact same mechanical model as the SEA, but with much lower physical stiffness and about half the dynamic mass. The performance characteristics should be comparable to the SEA, and be dissimilar in a predictable fashion.

Bandwidth tests of the SEA were implemented by clamping the output shaft and measuring the applied force, while commanding a sine wave force input of increasing frequency. The motor must move some amount to preload the springs, so the desired force will be applied to the output shaft. The inertia of the motor and the software proportional gain, along with any torque limitations, will determine the upper frequency limit that is achievable by the actuator. Because of the spring cancellation force term in the software controller, the system can be estimated as a damped mass-spring system, with damping and stiffness determined by the $\mathrm{P}$ and D software gains. Therefore, physical spring stiffness changes should not affect the performance of the system. However, stiffer springs perform better when torque limits of the motor are reached.

The AMASC has higher damping than the SEA, because a lower commanded damping ratio resulted in very large oscillations at the natural frequency, interfering with our tests. The consequence of this higher damping is a lower peak on the resulting Bode plot. The results of a physical test and a software simulation are shown in Figure 17(b), 
For all plots, $\mathrm{K}_{\mathrm{eff}}=5000$
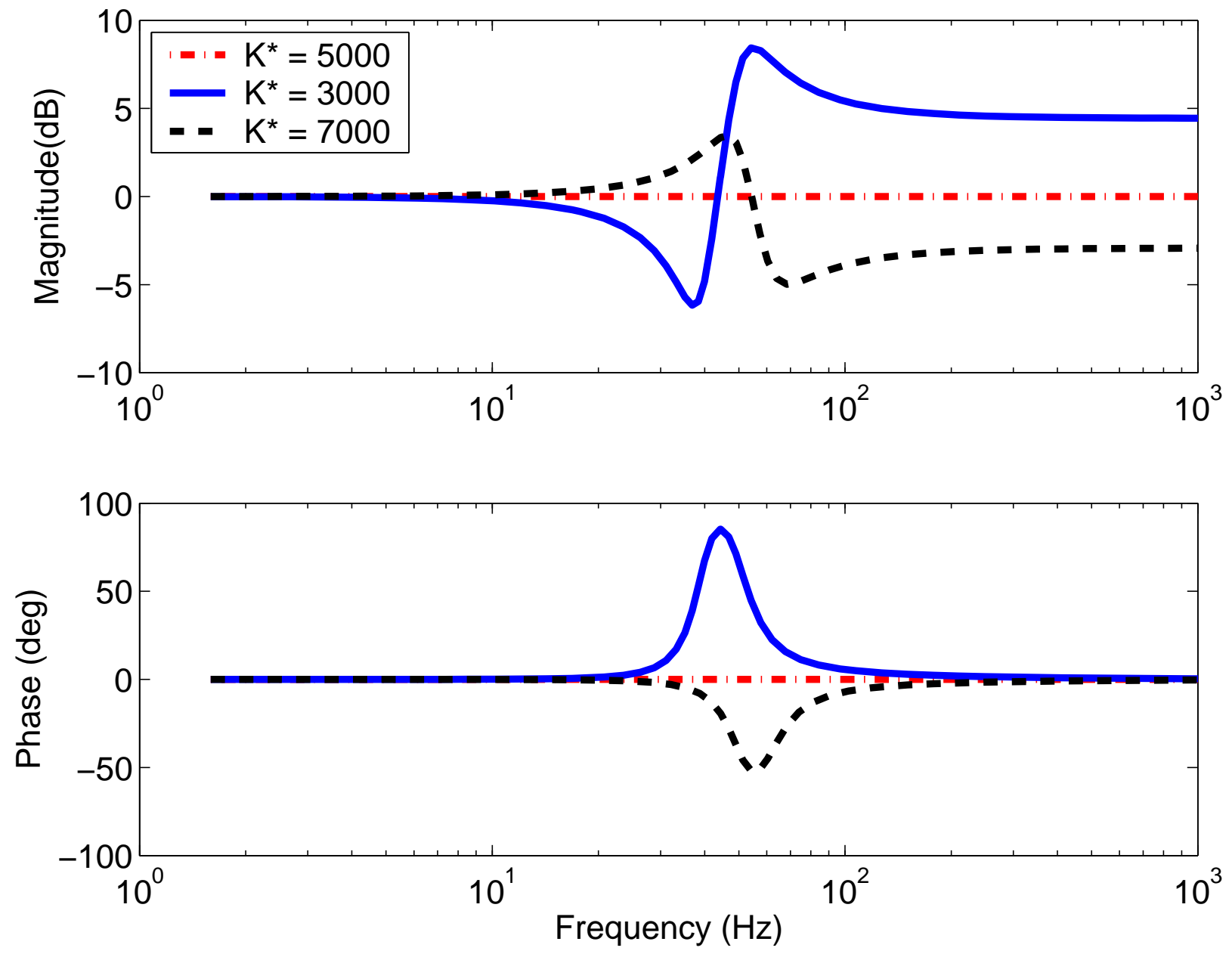

Figure 14: Impedance plot of the simulation. The system cannot keep up at high frequencies when improperly adjusted, but has no bandwidth limit when the system is tuned properly. 


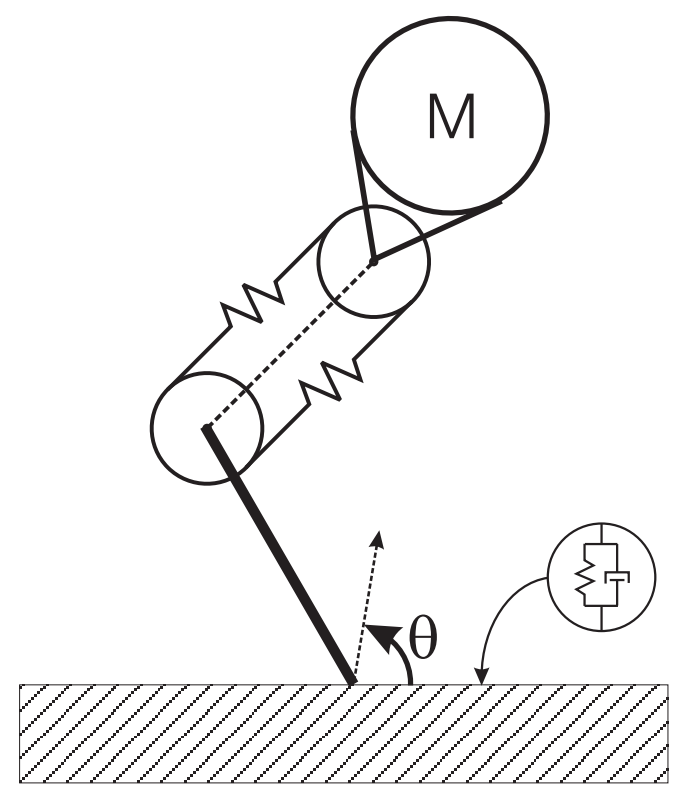

Figure 15: Hopping robot with the AMASC inserted as the knee spring.

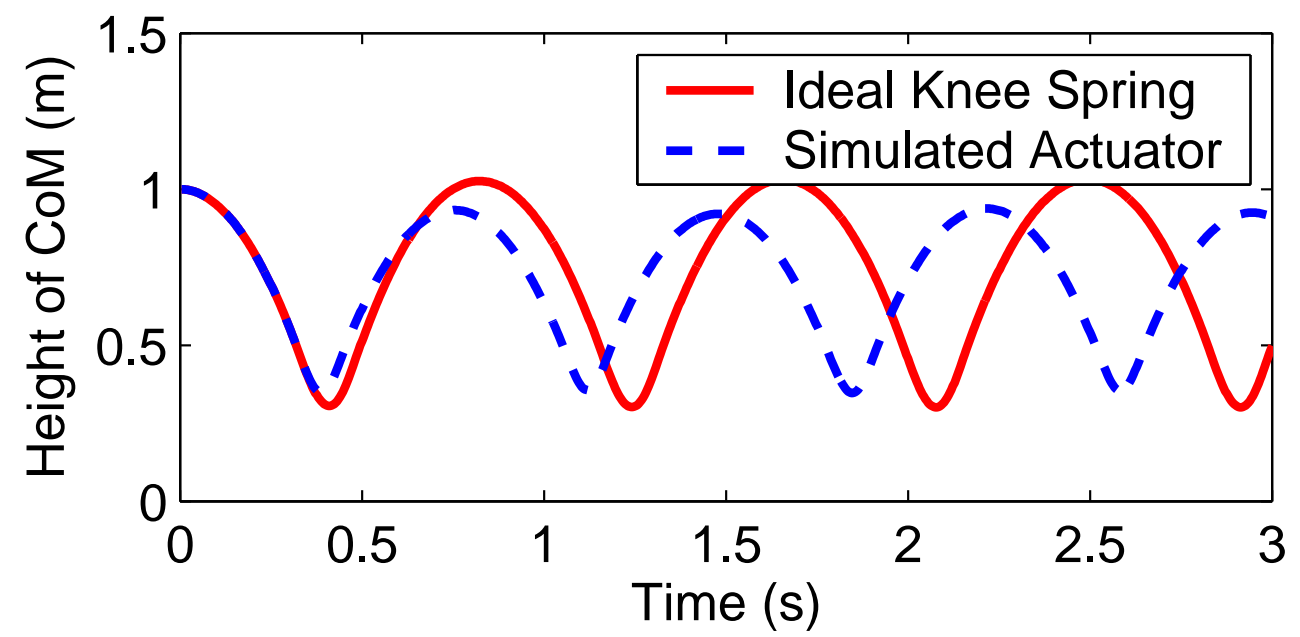

Figure 16: Comparison of hopping robot behavior with an ideal knee spring and a simulation of the AMASC as the knee spring.. 


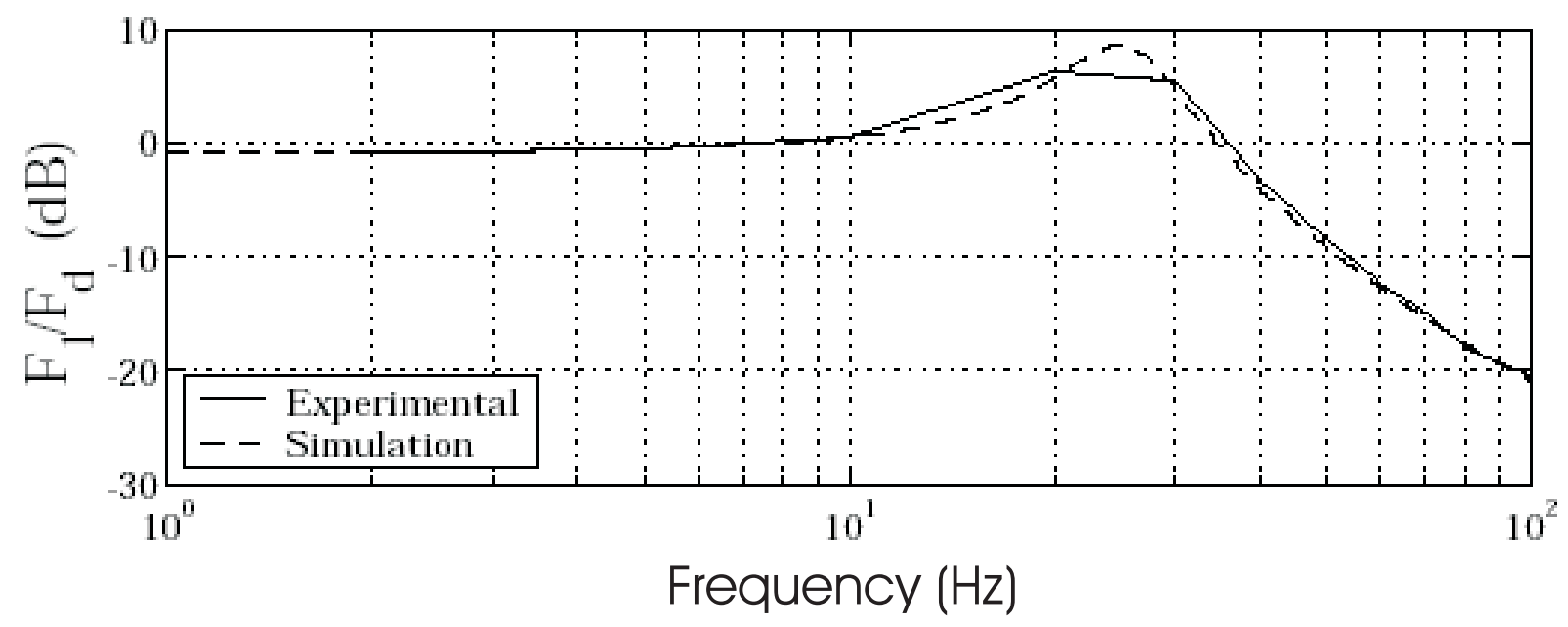

(a) Series elastic Actuator

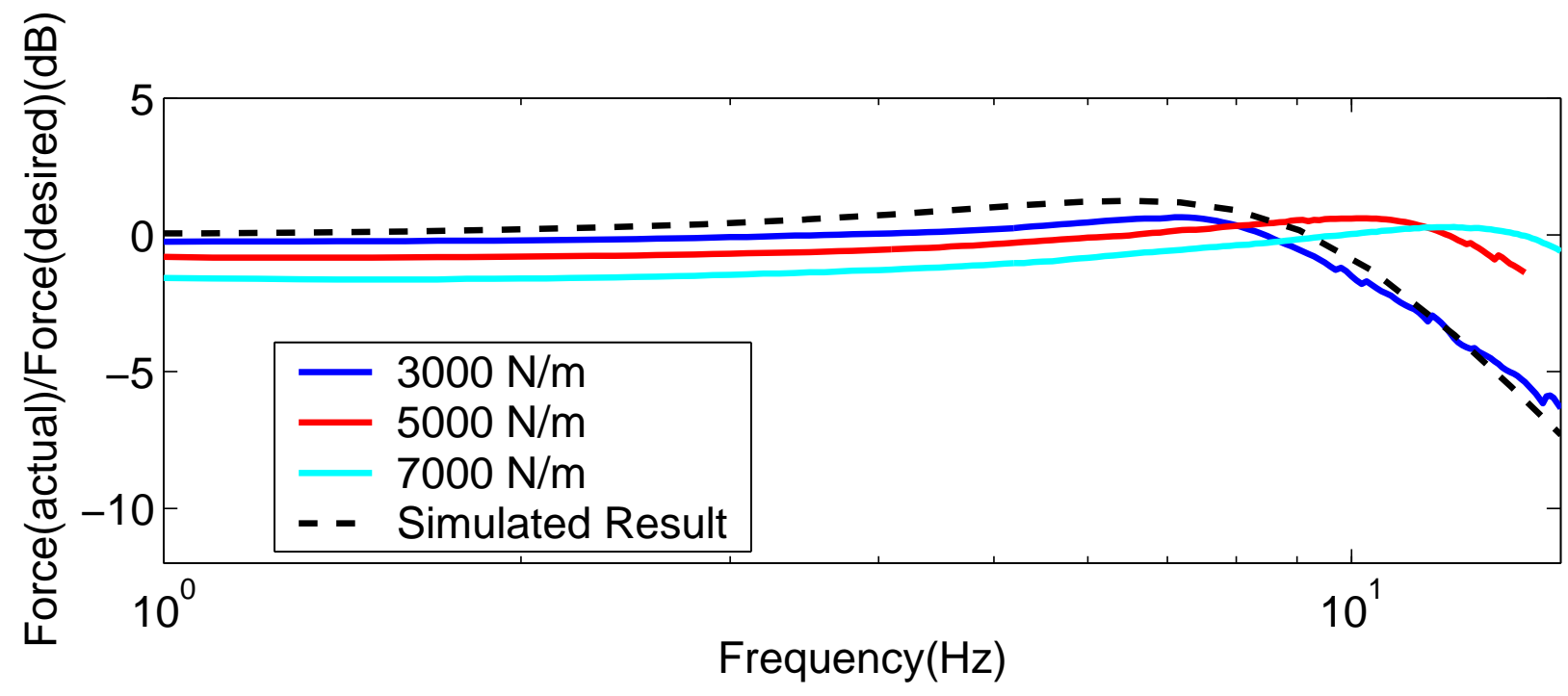

(b) AMASC

Figure 17: Comparison of force control bandwidth between the Series elastic actuator and the AMASC 
and can be compared to a frequency plot of the SEA in Figure 17(a) from David Robinson's Ph.D. Thesis [20]. The structure of each graph, whether from simulation or from data, is the same. The data for the AMASC shows different peak values for different amplitudes of input excitation, which should not occur for a linear system. It is most likely due to the nonlinearity of the knee spring; while it is nearly linear, that is only an approximation of the real system. The different peak value for the AMASC and the SEA may be attributed to different motor mass and different software proportional gains.

\subsection{The AMASC as a Manipulator}

A number of researchers have built mechanisms with variable compliance[34, 35, 36] for manipulation tasks. Most of these mechanisms use antagonistic motor pairs, which must exert forces both to precompress the springs and to move the joint. The required motor size and mass for such an antagonistic system is much larger than the AMASC, which uses one small motor with an electric brake to change the precompression (or pretension) of the springs. Any forces applied by the set-point motor are transmitted directly to knee torque, and do not need to overcome the opposing force of an antagonistic motor.

The stiffness function of other variable compliance mechanisms can be measured, but not chosen; the opposing springs tend to be a discrete component such as a coil spring or an air cylinder. The spring function is not a design freedom of the mechanism, as it is with the AMASC. In addition, the motors for all of these mechanisms must be placed at the actuated joint. This creates difficulty for a multi-jointed arm, where the base joint must lift all of the other joint motors as well as any payload. In contrast, the AMASC allows remote placement of the electric motor, while the structure and springs that are placed at the joint have low mass.

One difference between variable compliance designs for manipulation and designs for legged locomotion are the energy storage requirements. For locomotion, it is important to have springs that are large enough to store the energy of a running gait. For manipulation tasks, little or no energy is stored - the physical compliance is mainly important for safety or control system stability.

\section{Conclusions}

Physical compliance and mechanical energy storage are crucial for a successful running gait, while variable compliance is a useful control parameter for SLIP model running. The AMASC has mechanical energy storage, tunable compliance, low friction, and zero backlash. Within its range of mechanical compliance adjustment, the AMASC has virtually no bandwidth limitations and is similar to an ideal SLIP. Based on the results presented, the concepts embodied in the AMASC prototype could result in an effective actuation method for highly dynamic legged locomotion.

\subsection{Future Work}

There are still unanswered questions for further investigation. For example, we would like to determine what stiffness range is required for robust running on various surfaces, the necessary rate of stiffness adjustment, and the desired spring function. There may be benefits associated with mechanical damping that we have yet to explore. The morphology of the leg may have importance, with reasons for choosing certain link lengths between the joints of an articulated leg, or reasons to choose a purely prismatic leg.

There are also various improvements in the mechanical design that would result in a closer match to the mechanical model or reduce weight and complexity. The opposing block-and-tackle mechanism currently used as the power transmission involves many pulleys, which introduce significant friction. While the transmission friction does not affect the high-frequency response of the system, and can be overpowered by the software control system so it does not affect low-frequency behavior, it does demand a larger power drain than is strictly necessary. The spring set-point adjustment currently must apply force to stretch and release the spring as it adjusts; an additional spring can be placed in series that will assist the motor in making adjustments, and reduce its size and power requirements. Finally, further development could potentially reduce the overall complexity of the AMASC, without changing the behavior.

The computer simulation of the AMASC is relatively simple and accurate. Simulation of the running robot, however, is significantly more complex, but does not yet explore the desired range of behaviors and control. The ground surface should be modeled to more accurately represent real world surfaces. A more convenient control 
interface and graphical representation would make analysis much easier. We hope to use a more recent, refined simulator in future work.

The running controllers used in our simulation were attained almost directly from Marc Raibert's book[33]. There is further literature in both robotics and biomechanics that addresses the issue of efficient SLIP model running, and more complex controllers may allow better control of running. Our future work will focus on researching and creating controllers that utilize the benefits of the AMASC for SLIP model running.

\section{Acknowledgments}

This work is supported in part by an NSF Graduate Fellowship held by the first author and by the Robotics Institute of Carnegie Mellon University 


\section{References}

[1] William J. Schwind and Daniel E. Koditschek. Characterization of monopod equilibrium gaits. In Proceedings of the 1997 IEEE International Conference on Robotics and Automation, pages 1986-1992, Albequerque, New Mexico, April 1997.

[2] R. Blickhan. The spring-mass model for running and hopping. Journal of Biomechanics, 22(11/12):1217-1227, 1989.

[3] Thomas A. McMahon and George C. Cheng. The mechanics of running: How does stiffness couple with speed? Journal of Biomechanics, 23:65-78, 1990.

[4] Robert J. Full and Claire T. Farley. Musculoskeletal dynamics in rhythmic systems - a comparative approach to legged locomotion. In J. M. Winters and P. E. Crago, editors, Biomechanics and Neural Control of Posture and Movement. Springer-Verlag, New York, 2000.

[5] R. Blickhan and R. J. Full. Similarity in multilegged locomotion: Bouncing like a monopode. Journal of Comparative Physiology, pages 509-517, 1993.

[6] John R. Hutchinson, Dan Famini, Richard Lair, and Rodger Kram. Are fast-moving elephants really running? Nature, (422):493-494, 2003.

[7] Giovanni A. Cavagna, H. Thys, and A. Zamboni. The sources of external work in level walking and running. Journal of Physiology, 262:639-657, 1976.

[8] Giovanni A. Cavagna. Elastic bounce of the body. Journal of Applied Physiology, 29(3):279-282, 1970.

[9] Giovanni A. Cavagna, Norman C. Heglund, and C. Richard Taylor. Mechanical work in terrestrial locomotion: Two basic mechanisms for minimizing energy expenditure. American Journal Physiology, 233(5):R243-R261, 1977.

[10] T. A. McMahon. Mechanics of locomotion. The International Journal of Robotics Research, 3(2):4-28, 1984.

[11] Daniel P. Ferris and Claire T. Farley. Interaction of leg stiffness and surface stiffness during human hopping. The American Physiological Society, pages 15-22, 1997.

[12] Claire T. Farley, Han H. P. Houdijk, Ciska Van Strien, and Micky Louie. Mechanism of leg stiffness adjustment for hopping on surfaces of different stiffnesses. The American Physiological Society, pages 1044-1055, 1998.

[13] Daniel P. Ferris, Mickey Louie, and Claire T. Farley. Running in the real world: adjusting leg stiffness for different surfaces. In Proc. R. Soc. Lond., volume 265, pages 989-993, January 1998.

[14] Thomas A. McMahon and Peter R. Greene. The influence of track compliance on running. Journal of Biomechanics, 12:893-904, 1979.

[15] Thomas A. McMahon, Gordon Valiant, and Edward C. Frederick. Groucho running. Journal of Applied Physiology, 62:2326-2337, 1987.

[16] T. A. McMahon. The role of compliance in mammalian running gaits. Journal of Experimental Biology, 115:263$282,1985$.

[17] Jessica K. Hodgins and Marc H. Raibert. Adjusting step length for rough terrain. In IEEE Transactions on Robotics and Automation, volume 7, 1991.

[18] Claire T. Farley and Octavio Gonzalez. Leg stiffness and stride frequency in human running. Journal of Biomechanics, 29(2):181-186, 1995.

[19] C. T. Farley, J. Glasheen, and T. A. McMahon. Running springs: Speed and animal size. Journal of Experimental Biology, 185:71-87, 1993. 
[20] David William Robinson. Design and Analysis of Series Elasticity in Closed-Loop Actuator Force Control. PhD thesis, Massachusetts Institute of Technology, June 2000.

[21] Jerry Pratt, Peter Dilworth, and Gill Pratt. Virtual model control of a bipedal walking robot. In International Conference on Robotics and Automation, 1997.

[22] Jerry Pratt and Gill Pratt. Exploiting natural dynamics in the control of a planar bipedal walking robot. In Proceedings of the Thirty-Sixth Annual Allerton Conference on Communication, Control, and Computing, Monticello, IL, September 1998.

[23] Daniel Joseph Paluska. Design of a humanoid biped for walking research. Master's thesis, Massachusetts Institute of Technology, 2000.

[24] Jerry Pratt, Chee-Meng Chew, Ann Torres, Peter Dilworth, and Gill Pratt. Virtual model control: An intuitive approach for bipedal locomotion. The International Journal of Robotics Research, 2001.

[25] Antonio Bicchi, Giovanni Tonietti, Michele Bavaro, and Marco Piccigallo. Variable stiffness actuators for fast and safe motion control. In International Symposium on Robotics Research, 2003.

[26] J. De Schutter. A study of active compliant motion control methods for rigid manipulations based on a generic scheme. In IEEE, pages 1060-1065, 1987.

[27] Steven D. Eppinger and Warren P. Seering. Understanding bandwidth limitations in robot force control. In IEEE International Conference on Robotics and Automation, pages 904-909, 1987.

[28] Dale A. Lawrence. Actuator limitations on achievable manipulator impedance. In IEEE International Conference on Robotics and Automation, pages 560-565, 1989.

[29] David J. Bennett. What are the advantages of variable stiffness control? In IEEE Engineering in Medicine and Biology, pages 86-87, December 1992.

[30] Y. Matsuoka and R. D. Howe. Hand impedance change during learning of a novel contact task. In World Congress on Medical Physics and Biomedical Engineering, 2000.

[31] William T. Townsend and J. Kenneth Salisbury. Mechanical bandwidth as a guidline to high-performance manipulator design. In IEEE, pages 1390-1395, 1989.

[32] Nicholas P. Chironis. Mechanisms, Linkages, and Mechanical Controls, chapter Types of Noncircular Gears, pages 241-244. McGraw Hill, 1965.

[33] Marc Raibert. Legged Robots That Balance. MIT Press, Cambridge, Mass., 1986.

[34] Kirsten F. Laurin-Kovitz, J. Edward Colgate, and Steven D. R. Carnes. Design of components for programmable passive impedance. In Proceedings of the IEEE International Conference on Robotics and Automation, pages 1476-1481, April 1991.

[35] James K. Mills. hybrid actuator for robot manipulators: design, control and performance. In Proceedings of the IEEE International Conference on Robotics and Automation, pages 1872-1878, 1990.

[36] Toshio Morita and Shigeki Sugano. design and development of a new robot joint using a mechanical impedance adjuster. In IEEE International Conference on Robotics and Automation, pages 2469-2475, 1995. 